\title{
A Two-level Control Strategy with Fuzzy Logic for Large-scale Photovoltaic Farms to Support Grid Frequency Regulation
}

\author{
Nguyen Gia Minh Thao* and Kenko Uchida \\ Department of Electrical Engineering and Bioscience, Waseda University, Shinjuku-ku, Tokyo 169-8555, Japan.
}

\begin{abstract}
This study proposes a two-level coordinated control strategy with fuzzy logic for appropriately adjusting the total active power supplied to a grid by large-scale photovoltaic (PV) farms in order to regulate grid frequency. For a solar farm, the strategy includes a central coordinating controller and many local controllers at PV power assemblies, treated as agents. In detail, the central controller uses a frequency regulation module based on a new automatic-tuning fuzzy-logic controller scheme to compute the appropriate reference values according to the total power needed. Then, the individual reference value for each local controller is determined. Each local controller governs all power-electronic converters installed at the PV agent to inject power into the grid according to the individual reference value received. Additionally, each local controller uses an algorithm to manage the state-of-charge of the battery bank installed at the agent so that it remains in the safe range of $20-80 \%$ while operating and close to the desired idle value of $50 \%$ at the steady state. Besides, a special control mode is developed and integrated into the overall strategy to aid rapid recovery of the grid frequency under emergency conditions. Numerical simulations demonstrate that the suggested strategy has the good response in terms of injecting an appropriate amount of power into the grid to regulate the frequency deviation into acceptable ranges of $\pm 0.2(\mathrm{~Hz})$ in the transient state and $\pm 0.05(\mathrm{~Hz})$ at the steady state, even when the weather conditions (solar radiation, air temperature), AC system load, and important control parameters of the grid suddenly change. Furthermore, the effectiveness in improving the grid-frequency stabilization by using the proposed strategy is validated within a four-area power system, where four PV farms are connected and the operating parameters of the grids at the areas are fairly different.
\end{abstract}

Keywords: Coordinated control strategy, fuzzy logic controller, grid frequency regulation, large-scale PV farm, battery state-of-charge control, multi-area power system.

\section{Nomenclature}

$P \quad$ Physical value of active power, in Watts

$\beta \quad$ Value expressed in per-unit system of $P$

$\delta \quad$ Ratio value expressed in percent

$j \quad$ Index of local agent in a PV farm

$k \quad$ Present step of sampling time in control

\section{Superscripts}

* Reference value

Meas Measured actual value

Pre Predicted value

Min Minimum value

Max Maximum value

TrS Value computed in the transient state

StS Value computed at the steady state

Diff Difference value

\section{Introduction}

In recent years, large-scale PV solar farms have been widely used to inject active power into electric grids [1]-[4]. Nonetheless, large-scale solar farms, consisting of many local small PV power agents, can cause the grid frequency

\footnotetext{
This work is a significant modification and improvement over the study in our brief conference version [14].

* Nguyen Gia Minh Thao is the corresponding author.

E-mail: thao@uchi.elec.waseda.ac.jp $\quad$ Fax: +81-3-5273-9507 kuchida@uchi.elec.waseda.ac.jp
}

to deviate beyond the acceptable standard range of \pm 0.2 $(\mathrm{Hz})$. Moreover, in the case of multi-area power systems with many connected large-scale PV solar farms, the distortion in grid frequency will worsen sharply without a suitable and efficient coordinated control strategy. As well, energy storage systems (ESSs) have been broadly used in real large-scale PV farms to markedly enhance the robustness of farm performance in the face of unpredicted changes in weather condition. According to [5], an optimal design for solar farms with ESS is also necessary to maximize overall revenue in cases where grid-connected PV farms are considered as major components in a real energy market. Hence, the problem of regulating grid frequency in a power system that encompasses large-scale PV farms that use battery banks to manage energy needs to be examined and resolved satisfactorily. Toward resolving this issue, some control strategies have been proposed in [1]-[4],[6]-[12].

More specifically [1]-[3] describe a coordinated control method based on fuzzy logic for PV-diesel hybrid systems without battery banks. This method has been proposed for regulating grid frequency. It employs two 49-rule fuzzy logic controllers (FLCs) to compute the reference value for the active power that should be delivered to the grid. Nonetheless, in some cases, such as when the solar radiation decreases a large amount and the system load increases a large amount, a PV system without battery banks or another ESS may not supply sufficient power to the grid to allow the frequency to be stabilized. As shown in [4],[6], grid-connected PV systems with battery banks for managing energy have been used to control the grid frequency. 
The fuzzy-based coordinated strategy described in [4] has been evaluated within the test case of a three-area power system where some PV farms are connected. In most of the studies mentioned above, the state-of-charge (SOC) values of battery banks installed at the PV farms have not yet been managed strictly to stay within the ratio value range of $20-80 \%$ recommended to ensure durability of the battery banks.

As presented in [7], a proportional main controller and a double-layer capacitor for a grid-connected PV system have been applied to stabilize the grid frequency. The control approach not only has a relatively simple structure but also allows fine response. However, impacts of AC system loads in the grid were not examined deeply in [7]. Furthermore, a dynamic frequency-control technique that uses ultracapacitors as the major ESS to ameliorate the inertia of an isolated power grid on a French island, has been proposed in [8]. This technique well stabilized the grid frequency in the studied case, which involves a high penetration of PV solar and wind generators in an isolated grid. In addition to these systems, a frequency control technique based on a power curtailment algorithm using Newton quadratic interpolation (NQI) for grid-connected PV systems without ESS has been introduced and appraised in [9]. The main idea of the method in [9] is to tune frequently the operating point of the PV arrays so as to achieve the desired power amount. This scheme has not yet been tested within a large-scale PV farm that includes many local grid-connected solar agents.

The researchers in [10] have presented an online tuning approach that uses a combination of fuzzy logic and particle swarm optimization (PSO) to tune the design parameters of a proportional-integral controller. The goal of this intelligent control approach is to regulate the frequency of a typical AC microgrid, where some PV panels, wind turbine generators, ESSs, and fuel cells are connected. In another study, to address the issue of regulating both grid frequency and voltage for the distributed power generation, a model-free method based on the adaptive neuro-fuzzy inference system (ANFIS) has been introduced [11]. This method can well control the grid frequency and voltage even when there are unpredicted changes to the system load and the line parameters of the grid. The efficacy of the proposed ANFIS is dependent on the training process used to determine the parameters that determine the structure of the ANFIS, but this process often requires a larger amount of calculation and suitable databases.

In [13], a prior study by the present two authors showed a simple single-level control method suitable for a small PV power agent, but no coordinated control strategy was proposed for a large-scale PV farm. In addition, the tolerable range of grid frequency deviation at the steady state used in [13] was $\pm 0.075(\mathrm{~Hz})$, which is $50 \%$ larger than the often-used range of \pm 0.05 (Hz). A coordinated strategy for PV farms was presented initially in [14]. However, some other crucial issues have not been considered and evaluated, including: (i) special control for recovering rapidly the grid frequency in emergency conditions, (ii) supervision of the difference between the reference signal and the actual value of total power supplied from a PV farm, and (iii) performance within a multi-area power system with many
PV farms connected. As well, the algorithm that determines whether the grid is operating in the transient or steady state is simple and not highly accurate, which has a detrimental effect on the SOC management of battery banks in the PV farm. Lastly, the output scaling factors of the 49-rule FLCs in [13] and [14] are fixed values that are chosen manually, which may noticeably impair the ability of the control strategies to cope with large uncertainties in weather conditions and grid parameters.

Hence, in this paper, the above three issues (i, ii, iii) are studied carefully to resolve them thoroughly; moreover, the algorithm that determines the operating state of the grid is made significantly more accurate, which can better regulate the SOC of battery banks in the PV farm. Additionally, a modified 49-rule FLC scheme is employed in the central controller. The output scaling factor under this scheme is now adjusted automatically by a new complementary 6-rule FLC proposed here. This means the output scaling factor of the proposed FLC scheme is an online changeable value, instead of being a fixed value as in [13]-[14]. The ultimate objective of using this is to contribute to a substantial enhancement of the performance and adaptability of the suggested coordinated strategy. In addition, the structure of the proposed control strategy is much improved by new and useful features (described by Figs. 4 and 8 in Section 3) that were not present in the brief version shown in [14]. Furthermore, many illustrative diagrams, detailed explanations, test cases and simulation results are newly presented here.

The proposed coordinated strategy with fuzzy logic to support stabilization of the grid frequency for large-scale solar farms that use battery banks has the following key aims.

a) The total active power delivered from a PV farm to the grid should be tuned to ensure that the frequency is within a tolerable range. Two acceptable ranges for frequency deviation are $\pm 0.2(\mathrm{~Hz})$ for the transient state and $\pm 0.05(\mathrm{~Hz})$ at the steady state, where the nominal frequency is $60(\mathrm{~Hz})$.

b) At each local PV agent of the farm, the SOC of the battery bank should be maintained in the safe ratio range of $20-80 \%$ during the operating time to enhance the durability of the battery bank. Moreover, the steady-state SOC of the battery bank should be governed to an idle ratio value of $50 \%$ so long as the grid frequency deviation is in the small range of $\pm 0.05(\mathrm{~Hz})$. This function is intended to guarantee that the battery bank will have sufficient capacity for promptly supplying or absorbing energy as needed at the next operation time.

c) A control mode to regulate immediately the grid frequency deviation under emergency conditions should be designed, and its proper operation should be ensured.

d) Moreover, details of the efficacy of the suggested strategy within a four-area power system with four large-scale PV farms connected also are examined and evaluated.

e) The four objectives given in a), b), c) and d) of the strategy need to be verified as robust against unexpected changes in weather condition, AC system load, and crucial parameters of the automatic generation control (AGC) for the synchronous generator used in the grid. 
To meet all the objectives above, the proposed strategy for a PV solar farm is designed according to a two-level control structure as follows. At the lower level, initially, all local controllers at PV agents send key data from the agents to a central coordinating controller at the upper level. After that, the central controller, utilizing a frequency regulation module based on a novel automatic-tuning FLC scheme, determines the appropriate individual reference value of active power for the local controller at each PV agent in the farm. Finally, at the lower level, each local controller directly manages all the DC-DC converters connected to the PV arrays, a bidirectional DC-DC charger connected to a battery bank, and a three-phase DC-AC inverter in order to deliver the active power to the grid strictly according to the reference value obtained from the central controller. In addition to this, a control algorithm for governing SOC of the battery bank in both the transient and steady states is employed in the local controller at each PV agent. This control is intended to extend the life of the battery bank.

\section{The Demonstrative Large-scale PV Farm}

\subsection{Description of grid-connected large-scale PV farm}

A schematic diagram of the demonstrative grid-connected large-scale PV farm considered in this paper is shown in Fig. 1(a). The solar farm consists of a central coordinating controller and $N$ local PV power agents; each local agent comprises a local controller, four PV arrays and a battery bank. As shown in Fig. 1(b), each array has $n_{\text {Strings }}$ PV strings connected in parallel, and each string includes $n_{P V \text { panels }}$ solar panels connected in series. Hence, the total nominal output power $P_{P V \text { only,j }}^{\text {Rated }}$ of the four PV arrays in each local agent is computed by (1). The DC-DC converter used for each the PV array can be regarded as a non-inverting buck-boost converter, as described in [15]. The nominal capacity of the battery bank is $80 \%$ of the total nominal output power of the four PV arrays in the local agent, giving $P_{B a t, j}^{\text {Rated }}=80 \% \times P_{P V o n l y, j}^{\text {Rated }}$. Moreover, the battery bank can supply power (i.e., discharge) or absorb power (i.e., charge) via the bidirectional charger based on a half-bridge buck-book converter [4],[16]. The output active power of each local PV agent is supplied to the three-phase AC bus via a DC-AC inverter. All six power-electronic converters of each agent are driven by the local controller. Also, all local controllers and the central controller can exchange and transmit their key data together via a Common Industrial Protocol (CIP) such as DeviceNet [17] or EtherNet/IP [1],[4].

$$
P_{P V o n l y, j}^{\text {Rated }}=\sum_{i=1}^{4} P_{P V i, j}^{\text {Rated }}=\left[\left(P_{\text {panel }}^{\text {Rated }} \times n_{P \text { Vpanels }}\right) \times n_{\text {Strings }}\right] \times 4
$$

Furthermore, to focus our study on frequency deviation, it is assumed that the electric grid can be modeled as one or more synchronous generators, including speed governors. This is the so- called AGC scheme, shown in Fig. 2(a) and described in [18]. The AC system load is connected parallel to the $\mathrm{AC}$ bus.

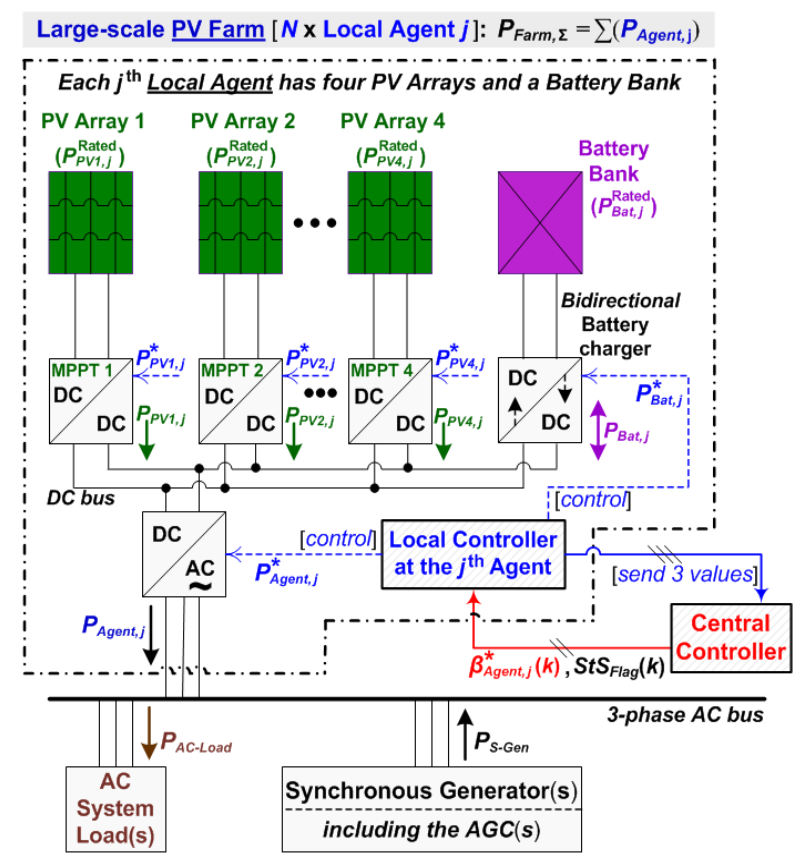

(a) Diagram of grid-connected large-scale PV farm

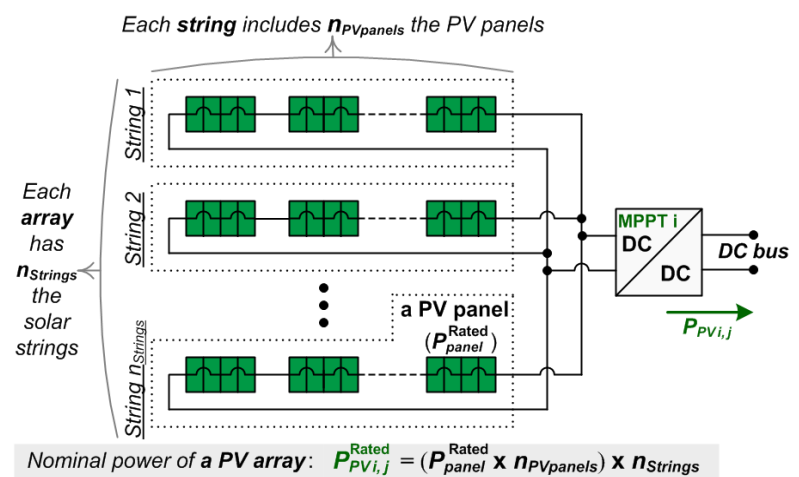

(b) Structure of a PV array installed at a local agent

Fig. 1. Schematic diagram of grid-connected large-scale PV farm utilized in this study.

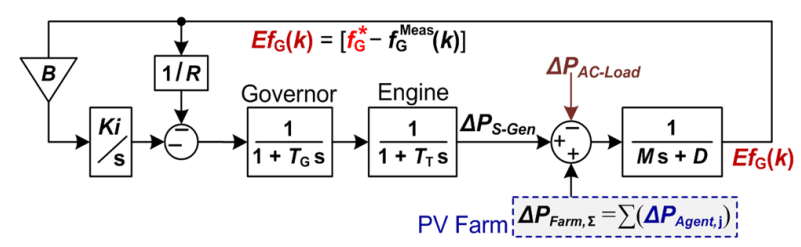

(a) Model of the speed governor (AGC) for a synchronous generator used in simulation

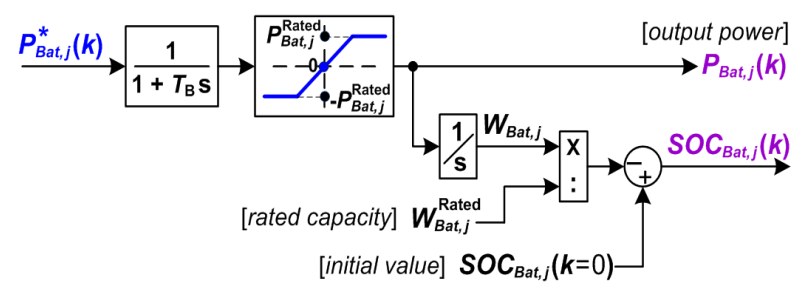

(b) Simplified model of a battery bank used in simulation

Fig. 2. AGC for the generator and battery bank seen in Fig. 1. 
According to [19] and [20], the multi-string PV power converter topology provides major advantages, combining the advantages of the string PV converter and central PV converter topologies. These advantages include low installment cost, high effectiveness in capturing energy via PV via using separate maximum power point tracking (MPPT) algorithms, optimal real-time supervision for solar energy systems, and very good compatibility for large-scale PV power farms. In addition to these advantages, commercial products that use the multi-string converter topology in real PV systems have been introduced and become popular. In this study, the multi-string DC-DC converter topology is used for each local PV power agent, as illustrated by Fig. 1(a).

\subsection{PV cell model}

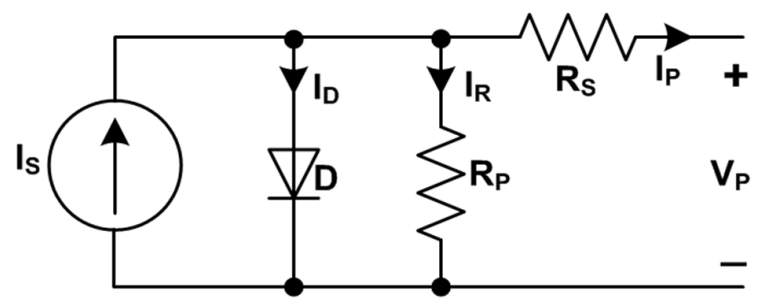

Fig. 3. Equivalent circuit for a PV solar cell [15].

According to [15],[21], the one-diode equivalent model of a PV solar cell is expressed in Fig. 3, (2) and (3).

$$
\begin{gathered}
I_{P}=I_{S}-I_{O}\left[\mathrm{e}\left(\frac{q\left(V_{P}+R_{S} I_{P}\right)}{a k T_{\text {Cell }}}\right)-1\right]-\left(\frac{V_{P}+R_{S} I_{P}}{R_{P}}\right) \\
\left\{\begin{array}{l}
I_{S}\left(T_{\text {Cell }}, G_{\text {So }}\right)=\left[I_{S C, n}+k_{T}\left(T_{\text {Cell }}-T_{n}\right)\right] \frac{G_{\text {So }}}{G_{n}} \\
I_{O}\left(T_{\text {Cell }}\right)=\frac{I_{S C, n}}{\mathrm{e}\left(\frac{q V_{\text {OC,n }}}{a k T_{\text {Cell }}}\right)-1}\left(\frac{T_{\text {Cell }}}{T_{n}}\right)^{3} \mathrm{e}\left[\frac{q E_{g}}{a k}\left(\frac{1}{T_{n}}-\frac{1}{T_{\text {Cell }}}\right)\right]
\end{array}\right.
\end{gathered}
$$

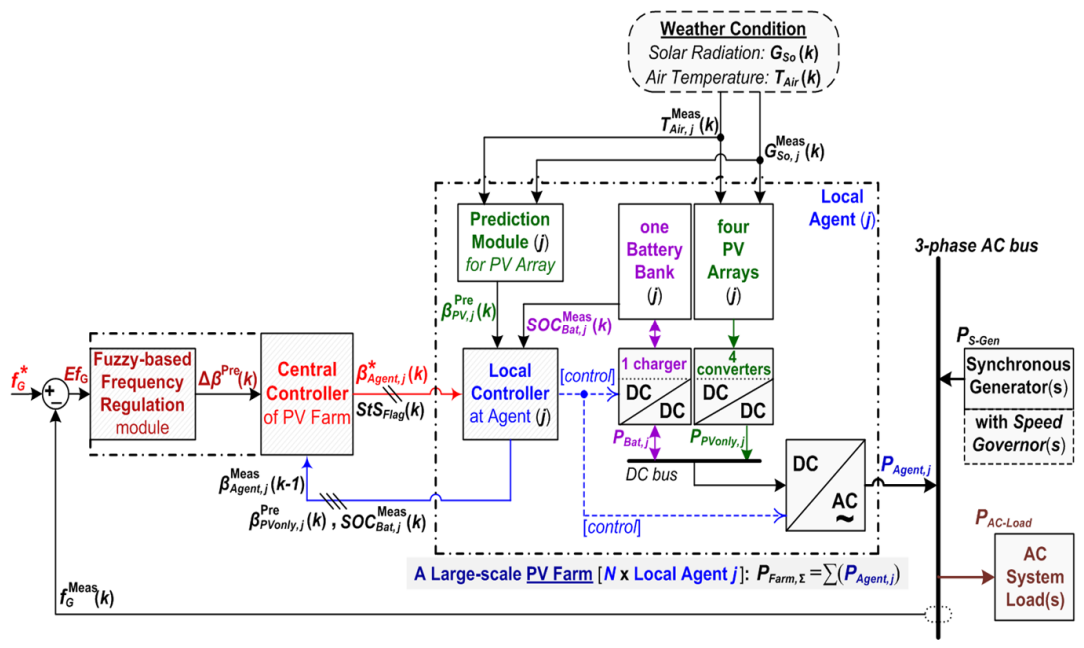

(a) Proposed strategy expressed in structure of grid frequency control
In the numerical simulations of this paper, a PV panel with a nominal power of $P_{\text {panel }}^{\text {Rated }}=250(\mathrm{~W})$ is used for the farm in Fig. 1, and its manufacturing parameters are given in Table 1.

Table 1. Nominal parameter values of 250-W PV panel [22]

\begin{tabular}{|l|c|c|}
\hline Parameter & Symbol & Value \\
\hline Nominal rated output power & $P_{\text {panel }}^{\text {Rated }}$ & $250 \mathrm{~W}$ \\
\hline Voltage at the MPP & $V_{\mathrm{MPP}}$ & $30.4 \mathrm{~V}$ \\
\hline Current at the MPP & $I_{\mathrm{MPP}}$ & $8.23 \mathrm{~A}$ \\
\hline Open-circuit voltage & $V_{\mathrm{OC}}$ & $38.1 \mathrm{~V}$ \\
\hline Short-circuit current & $I_{\mathrm{SC}}$ & $8.91 \mathrm{~A}$ \\
\hline $\begin{array}{l}\text { Number of cells in series, } \\
\text { parallel }\end{array}$ & $N_{\mathrm{S}}, N_{\mathrm{P}}$ & 60,1 \\
\hline
\end{tabular}

\section{Proposed Fuzzy-based Coordinated Control Strategy}

A detailed schematic diagram of the proposed coordinated strategy with fuzzy logic is shown in Fig. 4(a). In the strategy, each local controller at a PV agent will send the predicted maximum power value of all PV arrays belonging to that agent as simulated by the prediction module $\beta_{P V \text { only }, j}^{\text {Pre }}(k)$, the measured actual SOC of the battery bank $S O C_{B a t, j}^{\mathrm{Meas}}(k)$ and the actual active power injected into the grid in the previous step $\beta_{\text {Agent }, j}^{\text {Meas }}(k-1)$ from the PV agent to the central controller for handling.

Also, from the reference value $f_{G}^{*}$ and measured actual value $f_{G}^{\text {Meas }}(k)$ of the grid frequency, the newly proposed automatic-tuning FLC scheme, which is integrated in the central controller, determines the amount of adjustment $\Delta \beta^{\text {Pre }}(k)$ needed to precisely update the reference value of total active power so that it is the amount needed for injection into the grid $\beta_{F a r m, \Sigma}^{*}(k)$. By using (11), the reference value of total power $\beta_{\text {Farm, },}^{*}(k)$ is calculated from the data values sent from all local PV agents in combination with $\Delta \beta^{\text {Pre }}(k)$. Then, the individual reference power value $\beta_{\text {Agent, } j}^{*}(k)$ for each PV agent is calculated

Fig. 4. Detailed schematic diagram of the proposed fuzzy-based coordinated-control strategy for a large-scale PV farm.

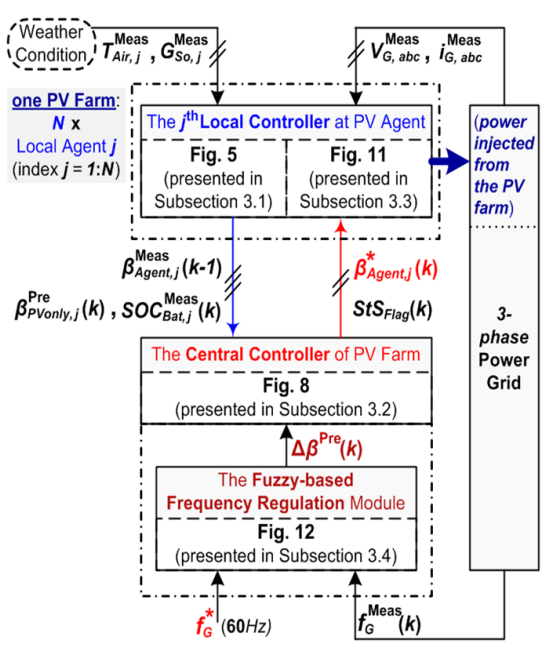

(b) Strategy re-expressed as design steps 
(see Steps B.4-B.5 below), and sent to the local controllers.

Last, the local controller appropriately controls the four DC-DC converters connected with four PV arrays, the bidirectional DC-DC charger used for the battery bank, and the three-phase DC-AC inverter. This is shown in Fig. 1(a). The effect of this governance is to inject the total active power of the local PV agent into the grid in a way that accurately provides the desired amount of active power $\beta_{\text {Agent }, j}^{*}(k)$ already specified by the central controller.

Fig. 4(b) presents the same information as in Fig. 4(a) in an alternative format to emphasize the details of the design steps of the proposed strategy. These steps are presented in Subsections 3.1-3.4. Subsection 3.1 contains the details of the prediction module implemented in the local controller at each PV agent; the key contents of this subsection are summarized in Fig. 5. Subsection 3.2 presents the steps executed in the central coordinating controller of the PV farm; the key features newly added in the proposed strategy are illustrated in Fig. 8. The next subsection gives operational details for the PV arrays and battery bank in each local solar agent; the flowchart of tasks is shown in Fig. 11. Lastly, Subsection 3.4 describes the design steps for the newly proposed automatic-tuning FLC scheme, which is utilized in the frequency regulation module of the central controller; the unique structure of the suggested FLC scheme is depicted in Fig. 12.

\subsection{Prediction module in each local controller}

The five steps shown in Fig. 5 are described here. The detailed structure of the prediction module installed at each local controller is shown in Fig. 6. In Fig. 6, the mathematical model of each PV panel is based on (2)-(3) and the manufacturing parameter values of a $250-\mathrm{W}$ PV panel (see Table 1 for the values used). The design parameter values of a PV panel can be easily found from the user guide or technical catalogue provided from the manufacturer.

Step A.1: According to [23],[24], the temperature of the PV cell (inside the PV panel) at each local agent can be estimated nearly correctly from the manufacturer's Nominal Operating Cell Temperature (NOCT) and the measured values of air temperature and solar irradiance as follows:

$$
T_{\text {Cell }, j}^{\mathrm{Pre}}(k)=T_{\text {Air }, j}^{\mathrm{Meas}}(k)+(N O C T-20) \times \frac{G_{S o, j}^{\mathrm{Meas}}(k)}{800}
$$

With the temperature of solar cell computed in (4) and the value of solar radiation as measured by the sensor, the output current of each PV solar cell can be estimated from (2). Then, the processes shown in Figs. 6 and 7 are implemented in a computer simulation to forecast the maximum power $\beta_{P V, j}^{\mathrm{Pre}}(k)$ of a PV array shown in Fig 1(b). As seen in Fig. 7, mathematical models of the conventional MPPT combined with a PI controller and a non-inverting buck-boost converter are obtained from [21],[15]. From these, the predicted maximum total capacity of all the four PV arrays in the local agent is calculated as $\beta_{P V o n l y, j}^{\text {Pre }}(k)=\sum_{i=1}^{4}\left(\beta_{P V i, j}^{\text {Pre }}(k)\right)$.

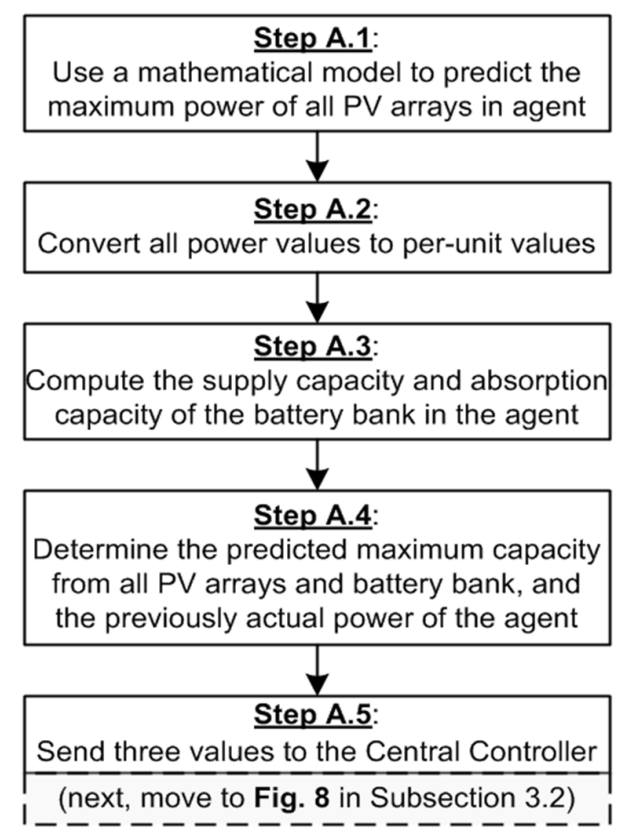

Fig. 5. Flowchart of five design steps presented in Subsection 3.1.

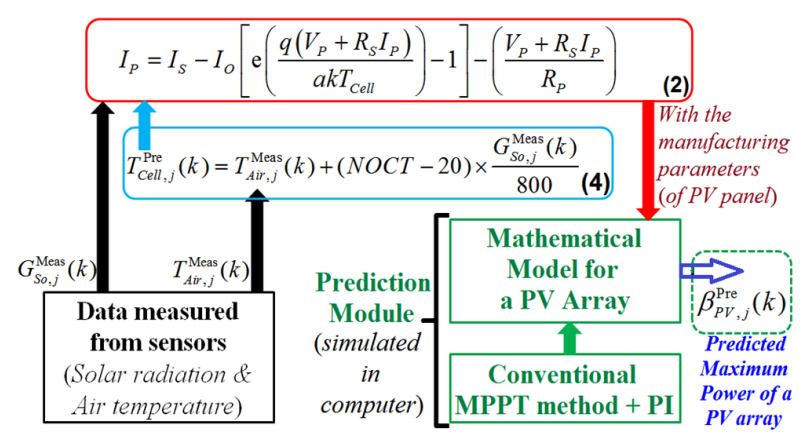

Fig. 6. Scheme of the prediction module in local PV agent.

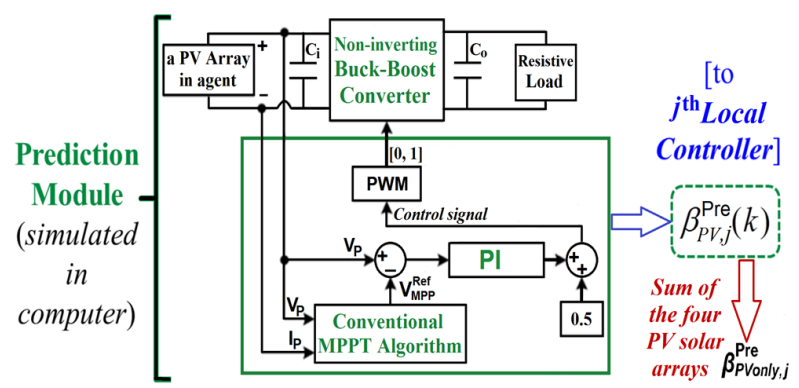

Fig. 7. Structure for implementing the conventional MPPT for a PV array inside the prediction module.

Step A.2: In this research, all the systematic parameters are converted to per-unit values (notated as p.u.) in order to simplify the design process of the suggested strategy in the three-phase grid with high power [25],[26]. A suitable base value, which is used for converting the power values in both the PV farm and the grid to per-unit values, can be chosen as the grid power capacity, using $P_{p . u \text {. }}^{\text {Base }}=P_{\text {Grid }}^{\text {Capacity }}$. Some 
typical per-unit values expressed as $\beta$ at each local PV power agent and in the PV farm are given in (5).

$$
\left\{\begin{array}{l}
\beta_{P V \text { only }, j}^{\text {Rated }}(k)=\frac{P_{P V \text { only }, j}^{\text {Rated }}(k)}{P_{p . u .}^{\text {Base }}} ; \beta_{\text {Bat }, j}^{\text {Rated }}=\frac{P_{\text {Bat }, j}^{\text {Rated }}}{P_{p . u .}^{\text {Base }}} \\
\beta_{P V \text { only }, j}^{\text {Pre }}(k)=\frac{P_{P V \text { only }, j}^{\text {Pre }}(k)}{P_{p . u .}^{\text {Base }}} ; \beta_{P V \text { only }, j}^{\text {Meas }}(k)=\frac{P_{P V \text { only }, j}^{\text {Meas }}(k)}{P_{p . u .}^{\text {Base }}}(5) \\
\beta_{\text {Agent }, j}^{*}(k)=\frac{P_{\text {Agent }, j}^{*}(k)}{P_{p . u .}^{\text {Base }}} ; \beta_{\text {Farm }, \Sigma}^{*}(k)=\frac{P_{F a r m, \Sigma}^{*}(k)}{P_{p . u .}^{\text {Base }}}
\end{array}\right.
$$

For example, in numerical simulations described in this paper, each array in Fig. 1(b) has ten PV strings connected in parallel, and each string consists of ten $250-\mathrm{W}$ solar panels connected in series. According to (1) with $n_{\text {Strings }}=10$ and $n_{P V \text { panels }}=10$, the total nominal output power of the four PV arrays in the local agent is $P_{P V \text { only, } j}^{\text {Rated }}=[(250 \mathrm{~W} \times$ $10) \times 10] \times 4=100(\mathrm{~kW})$. The nominal capacity of the battery bank is $80 \%$ of the total nominal output power of the four PV arrays in the agent, giving $P_{B a t, j}^{\text {Rated }}=80 \% \times 100$ $\mathrm{kW}=80(\mathrm{~kW})$. The PV farm is connected to a grid with a nominal power capacity of $6(\mathrm{MW})$, and this means that 1 (p.u.) corresponds to a normalized value of $P_{p . u \text {. }}^{\text {Base }}=$ $P_{\text {Grid }}^{\text {Capacity }}=6(\mathrm{MW})$. The first two values in (5) are computed as follows: $\beta_{P V o n l y, j}^{\text {Rated }}=P_{P V o n l y, j}^{\text {Rated }} / P_{p . u}^{\text {Base }}=100 \mathrm{~kW} / 6 \mathrm{MW}$ $=0.0167$ (p.u.), and $\beta_{B a t, j}^{\text {Rated }}=P_{B a t, j}^{\text {Rated }} / P_{\text {p.u. }}^{\text {Base }}=80 \mathrm{~kW} / 6$ $\mathrm{MW}=0.0133$ (p.u.).

Step A.3: At each local PV power agent, a battery bank is used to help efficiently manage the obtained electric energy and protect against unpredicted changes in weather. In this study, to allow focusing mainly on the proposed coordinated control strategy, the simplified model shown in Fig. 2(b) is used for the battery bank in MATLAB simulation. The power absorption and supply capacities of the battery bank in the transient state are calculated by (6) and (7), respectively.

$$
\beta_{B a t, j}^{\text {Pre,Absorb }}(k)=\left\{\begin{array}{l}
0, \text { if } S O C_{B a t, j}^{\mathrm{Meas}}(k)>S O C_{B a t}^{\mathrm{Max}}=80 \% \\
{\left[S O C_{B a t, j}^{\mathrm{Meas}}(k)-S O C_{B a t}^{\mathrm{Max}}\right] \times \beta_{B a t, j}^{\mathrm{Rated}}, \text { otherwise }}
\end{array}\right.
$$

$$
\beta_{\text {Bat }, j}^{\text {Pre,Supply,TrS }}(k)=\left\{\begin{array}{l}
0, \text { if } S O C_{B a t, j}^{\mathrm{Meas}}(k)<S O C_{B a t}^{\mathrm{Min}}=20 \% \\
{\left[S O C_{B a t, j}^{\mathrm{Meas}}(k)-S O C_{B a t}^{\mathrm{Min}}\right] \times \beta_{\text {Bat }, j}^{\mathrm{Rated}}, \text { otherwise }}
\end{array}\right.
$$

Step A.4: The predicted maximum capacity from all four PV arrays and the battery bank of each local PV power agent is shown in (8). The actual active power supplied to the grid in the previous time step by each PV agent is expressed in (9).

$$
\begin{aligned}
& \beta_{\text {Agent }, j}^{\text {Pre,Max,TrS }}(k)=\beta_{P V \text { only }, j}^{\text {Pre }}(k)+\beta_{\text {Bat }, j}^{\text {Pre,Supply,TrS }}(k) \\
& \beta_{\text {Agent }, j}^{\text {Meas }}(k-1)=\beta_{P V \text { only }, j}^{\text {Meas }}(k-1)+\beta_{\text {Bat }, j}^{\text {Meas }}(k-1)
\end{aligned}
$$

Step A.5: From each local controller of the PV agent in the solar farm, the three data values $\beta_{P V o n l y, j}^{\text {Pre }}(k), S O C_{B a t, j}^{\text {Meas }}(k)$, and $\beta_{\text {Agent }, j}^{\text {Meas }}(k-1)$ given by, respectively, Step A.1, (7) and (9), will be sent to the central coordinating controller of the PV farm for handling, as already shown in Fig. 4(a).

\subsection{Steps of execution in the central controller}

Fig. 8 shows the flowchart of six steps of execution in the central coordinating controller of the PV farm. The most important aspects are as follows. The reference signal of the total power supply needed from the PV farm, $\beta_{\text {Farm }, \Sigma}^{*}(k)$, is computed in Step B.2. The three operating states of the grid (emergency condition, transient state, and steady state) are determined and presented in Steps B.3-B.5. Steps B.3, B.4(a) and B.5 are particularly important parts of the proposed control strategy and are newly added here (cf. [13] and [14]).

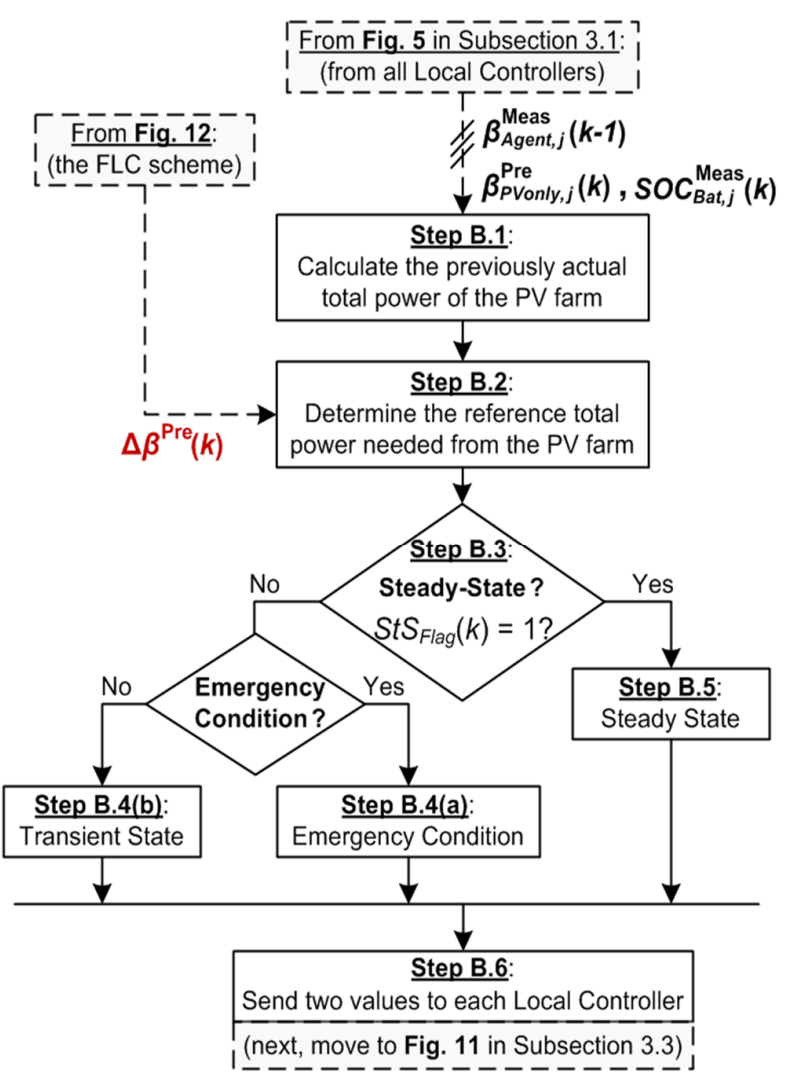

Fig. 8. Flowchart of design steps presented in Subsection 3.2.

Step B.1: The actual total power previously delivered to the grid $\beta_{F a r m, \Sigma}^{\text {Meas }}(k-1)$ of the solar farm is computed in (10).

$$
\beta_{\text {Farm }, \Sigma}^{\text {Meas }}(k-1)=\sum_{j=1}^{N} \beta_{\text {Agent }, j}^{\text {Meas }}(k-1)
$$

Step B.2: With the adjustment $\Delta \beta^{\text {Pre }}(k)$ to the power value as shown in Fig. 4 (see Fig. 12 of Subsection 3.4 for more details), the $k$ th desired value of total active power for the PV 
farm, consisting of all $N$ local solar agents, is computed by (11).

$$
\beta_{\text {Farm }, \Sigma}^{*}(k)=\beta_{\text {Farm }, \Sigma}^{\text {Meas }}(k-1)+\left[N \times \Delta \beta^{\text {Pre }}(k)\right]
$$

Here, the limiting value of $\beta_{F a r m, \Sigma}^{*}(k)$ will be expressed by (15) in Step B.3.

Step B.3: Determine the present operating state of the power system, and set the limits for the reference signal.

In this study, a short program is proposed for enhancing the accuracy of determining the present operating state (such as the transient or steady state) of the power system, as follows.

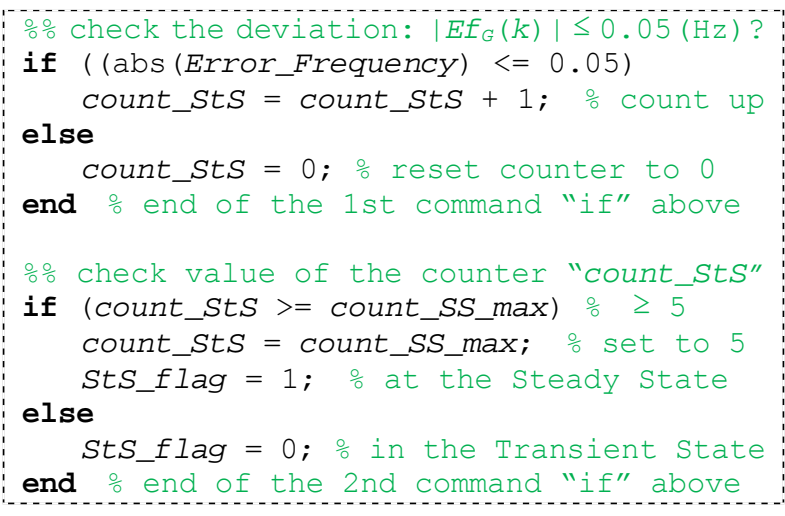

In this program, the variable Error_Frequency is the grid frequency error $E f_{G}(k)$ as shown in Figs. 2(a) and 4(a). The variable StS_flag is a flag to indicate the steady state when set. This is named $S t S_{\text {Flag }}(k)$ in Figs. 4 and 8. The variable count_SS_max is the threshold for the maximum number of times the frequency error $E f_{G}(k)$ will be checked. In this study, count_ss_max is set to 5, as shown in Fig. 9. The value of the count variable count_StS is increased when the frequency error $E f_{G}(k)$ is inside the very small range of $\pm 0.05(\mathrm{~Hz})$, and its value is reset to 0 when $E f_{G}(k)$ is outside that range. In fact, the above program is designed to prioritize careful determination of whether the power system is in a steady state. This improves the adaptability of the proposed FLC scheme and better regulates the SOC of battery banks during the steady state.

For example, as illustrated in Fig. 9, with the first case, of five consecutive checking times at points $n_{1}, n_{2}, n_{3}, n_{4}$, and $n_{5}$, it can be seen that the frequency error at the four points $n_{2}, n_{3}, n_{4}$, and $n_{5}$ is outside the range $\pm 0.05(\mathrm{~Hz})$. For the second case of five consecutive checking times, at points $r_{1}, r_{2}, r_{3}, r_{4}$, and $r_{5}$, the is outside the range $\pm 0.05(\mathrm{~Hz})$ at $r_{5}$. These observations mean that the power system in the above two cases is still in the transient state, so the indication flag $S t S_{\text {Flag }}(k)$ is set to 0 (i.e., false). For the third case of five consecutive checking times, at points $s_{1}$, $s_{2}, s_{3}, s_{4}$, and $s_{5}$, the frequency error at all five points is inside the range $\pm 0.05(\mathrm{~Hz})$; this means the power system is at the steady state. Therefore, the indication flag $\operatorname{StS}_{\text {Flag }}(k)$ is set to 1 (i.e., true) from the time of the checking point $S_{5}$. This checking process will continue to execute in this way to update the real-time value of $S t S_{\text {Flag }}(k)$, which will be used as an input for the proposed automatic-tuning FLC scheme given in Fig. 12.

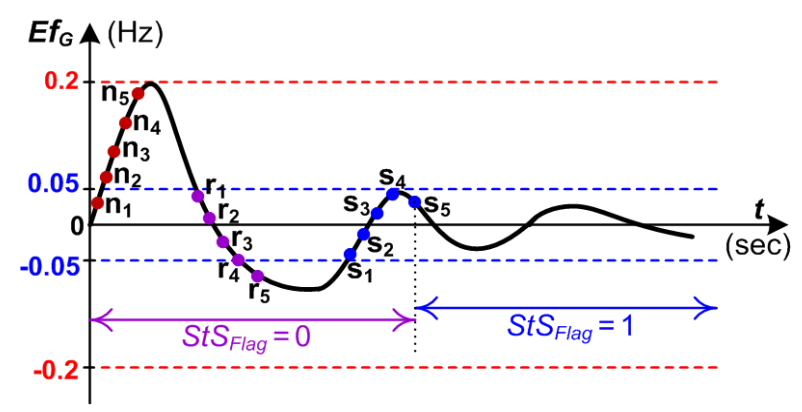

Fig. 9. Checking process of grid frequency deviation.

In [14], an abbreviated version of this paper, the grid frequency error was checked at only a single operating point when detecting the steady state; this means the particular value of the variable count_SS_max in the above program would be chosen as 1 . However, with this technique, the control system may not correctly determine whether the system is in a steady state at the check points $n_{1}, r_{1}, r_{2}, r_{3}$, and $r_{4}$. In fact, the power system at these points is still in a transient state. This drawback of a less strict check can cause the prior system to generate unsuitable reference signals in the PV farm. In fact, an appropriate value for the threshold variable count_SS_max is chosen manually according to the relative ratio between the time constant of grid frequency deviation and the sampling time used for control, where the control sampling time in this study is $T_{s-\text { Control }}=0.05(\mathrm{~s})$.

- Set limiting values for the reference signal $\beta_{F a r m, \Sigma}^{*}(k)$ $\beta_{B a t, j}^{\text {Pre,Supply,Trs }}(k)=\left\{\begin{array}{l}0, \text { if } S O C_{B a t, j}^{\text {Meas }}(k)<S O C_{B a t}^{\mathrm{Min}}=20 \% \\ {\left[S O C_{B a t, j}^{\text {Meas }}(k)-S O C_{B a t}^{\text {Min }}\right] \times \beta_{B a t, j}^{\text {Rated }}, \text { otherwise }}\end{array}\right.$

The predicted maximum total capacities of a local agent $\beta_{\text {Agent }, j}^{\text {Pre,Max,TrS }}(k)$ and of the PV farm $\beta_{\text {Farm }, \Sigma}^{\text {Pre,Max,TrS }}(k)$ in the transient state are computed in (13) and (14), respectively.

$$
\begin{gathered}
\beta_{\text {Agent }, j}^{\text {Pre,Max,TrS }}(k)=\beta_{P V \text { only }, j}^{\text {Pre }}(k)+\beta_{\text {Bat }, j}^{\text {Pre,Supply,TrS }}(k) \\
\beta_{\text {Farm }, \Sigma}^{\text {Pre,Max }, T r S}(k)=\sum_{j=1}^{N} \beta_{\text {Agent }, j}^{\text {Pre,Max }, T r S}(k)
\end{gathered}
$$

The value $\beta_{F a r m, \Sigma}^{\text {Pre,Max,TrS }}(k)$ expressed in (14) is taken as an upper limit for the reference signal $\beta_{F a r m, \Sigma}^{*}(k)$ already computed in (11), as shown by (15). Besides, in this paper, the PV farm is designed only to supply active power to the grid and cannot absorb power from the grid, so the desired total power of the PV farm, $\beta_{\text {Farm }, \Sigma}^{*}(k)$, must be a non-negative value.

$$
0 \leq \beta_{\mathrm{Farm}, \Sigma}^{*}(k) \leq \beta_{\mathrm{Farm}, \Sigma}^{\mathrm{Pre}, \mathrm{Max}, \mathrm{TrS}}(k)
$$


In numerical simulations, according to Step A.2 and (7), the maximum capacity of each local PV agent predicted by (13) is limited by $\beta_{\text {Agent }, j}^{\text {Pre,Max }} \leq[0.0167$ p.u. $+(100 \%-20 \%) \times$ 0.0133 p.u.] $=0.02734$ (p.u.). So, the predicted maximum total capacity of the PV farm shown in (14) and (15) is limited to $\beta_{F a r m, \Sigma}^{\text {Pre,Max,TrS }} \leq N \times 0.02734$ (p.u.), where 1 (p.u.) corresponds to $6(\mathrm{MW})$ of normalized power.

If the value of variable StS_flag in the above program is 0 (that is, if $\operatorname{StS}_{\text {Flag }}(k)=0$ as, illustrated in Fig. 9), then the system is determined as currently operating in the transient state. In that case, the central controller will move to Step B.4 below for its next execution (see Fig. 8). In contrast, if the value of variable StS_flag is 1 , the power system is regarded as presently operating at the steady state, and so, the central controller will ignore Step B.4 below and move directly to Step B.5 for the next execution.

\section{Step B.4:}

a. Special frequency control in the emergency condition

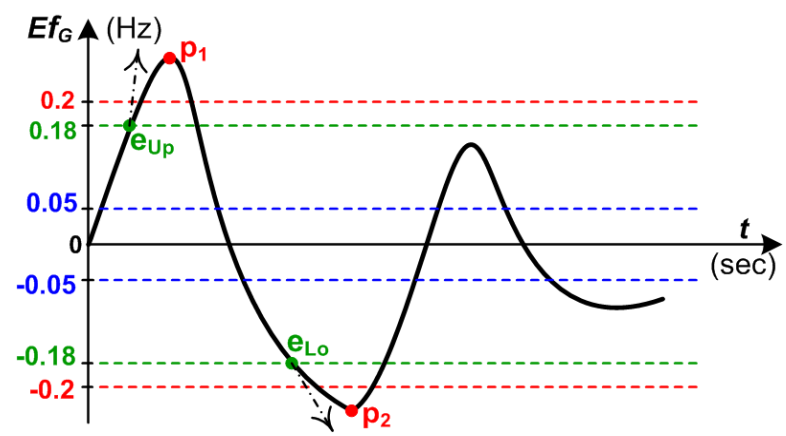

Fig. 10. Determination of emergency cases for frequency.

As shown in the upper part of Fig. 10, the first emergency condition for frequency control is defined as in (16).

$$
\left\{\begin{array}{l}
E f_{G}(k)=\left[f_{G}^{*}-f_{G}^{\text {Meas }}(k)\right] \geq \alpha_{\text {Limit }}=0.18 \\
\Delta E f_{G}(k)=\left[E f_{G}(k)-E f_{G}(k-1)\right] \geq \Delta \alpha_{\text {Limit }}=0.02
\end{array}\right.
$$

In (16), because $E f_{G}(k)+\Delta E f_{G}(k) \geq 0.18+0.02=0.2$, the grid frequency deviation $E f_{G}(k+1)=\left[f_{G}^{*}-\right.$ $\left.f_{G}^{\text {Meas }}(k+1)\right]$ is predicted for the next time step to exceed the upper acceptable limit of $0.2(\mathrm{~Hz})$. This may occur, for example, if the operating point of grid frequency deviation $E f_{G}(k)$ moves up rapidly from the currently acceptable point $e_{\mathrm{Up}}$ to the intolerable point $p_{1}$ (as in Fig. 10) when there is no special frequency control in place.

In this emergency case, according to [18] and Fig. 2(a), the power injected into the grid is currently too much larger than the demanded amount. To address this, each local PV agent in the solar farm should be controlled to operate at only $25 \%$ of its total power capacity (this means that only one of four PV arrays of each agent will be activated for operation), and the battery bank is set, with high priority, to absorb most of the energy provided from the PV arrays. The goal is to reduce sharply the power supplied to the grid from the PV farm, which would otherwise increase the frequency $f_{G}^{\text {Meas }}(k+1)$. This action aims to promptly reduce $E f_{G}(k+1)$. The individual reference value for each local agent is defined by (17).

$$
\begin{aligned}
& \beta_{\text {Agent }, j}^{*}(k)=\left[\beta_{P V \text { only }, j}^{\text {Pre }}(k) \times 25 \%\right]+\beta_{\text {Bat }, j}^{\text {Pre,Absorb }}(k) \\
& \beta_{B a t, j}^{\text {Pre,Absorb }}(k)=\left\{\begin{array}{l}
0, \text { if } S O C_{B a t, j}^{\text {Meas }}(k)>S O C_{B a t}^{\mathrm{Max}}=80 \% \\
{\left[S O C_{B a t, j}^{\mathrm{Meas}}(k)-S O C_{B a t}^{\mathrm{Max}}\right] \times \beta_{B a t, j}^{\mathrm{Rated}}, \text { otherwise }}
\end{array}\right.
\end{aligned}
$$

Additionally, as given in the lower part of Fig. 10, the second emergency condition for frequency control is defined by (19).

$$
\left\{\begin{array}{l}
E f_{G}(k)=\left[f_{G}^{*}-f_{G}^{\text {Meas }}(k)\right] \leq-\alpha_{\text {Limit }}=-0.18 \\
\Delta E f_{G}(k)=\left[E f_{G}(k)-E f_{G}(k-1)\right] \leq-\Delta \alpha_{\text {Limit }}=-0.02
\end{array}\right.
$$

In (19), because $E f_{G}(k)+\Delta E f_{G}(k) \leq-0.18-0.02=-0.2$, the grid frequency deviation $E f_{G}(k+1)=\left[f_{G}^{*}-\right.$ $\left.f_{G}^{\text {Meas }}(k+1)\right]$ at the next time is predicted to exceed negatively the lower threshold of $-0.2(\mathrm{~Hz})$. For example, the operating point of grid frequency deviation $E f_{G}(k)$ might move down quickly from the currently acceptable point $e_{\mathrm{Lo}}$ to the intolerable point $p_{2}$, as seen in Fig. 10, if there is no special frequency control in place.

In this emergency case, according to [18] and Fig. 2(a), the power injected into the grid will not be sufficient to meet the demand. Hence, each local PV agent in the solar farm should be forced to supply its maximum power capacity to the grid to thereby reduce the grid frequency $f_{G}^{\text {Meas }}(k+1)$, with the ultimate aim of swiftly boosting $E f_{G}(k+1)$. From this, the individual reference value for each PV agent is given by (20) for this case.

$$
\beta_{\text {Agent }, j}^{*}(k)=\left(1-\mu_{L, j}\right) \times \beta_{\text {Agent }, j}^{\text {Pre,Max }}(k)
$$

In (20), $\mu_{L, j}=5 \%$ is the percentage difference between the estimated total power loss and the nominal total capacity in each local PV agent. Section 5 describes the meaning more fully.

As expressed in (16) and (19), the value of $\alpha_{\text {Limit }}$ can be chosen as $90 \% \times 0.2=0.18$ and the value of its deviation $\Delta \alpha_{\text {Limit }}$ can be chosen as 0.02 to determine the thresholds for the two emergency conditions. The goal in setting these limits is to restrict, actively and in advance, large grid frequency distortions, which tend to occur around the upper and lower boundaries of the tolerable range of \pm 0.2 (Hz). If, instead, the value $\alpha_{\text {Limit }}$ is chosen to be larger than the threshold of 0.2 , the grid frequency deviation $E f_{G}(k)$ will surely leave the acceptable range of $\pm 0.2(\mathrm{~Hz})$ for a while; when it does so, the control system will need additional time to force $E f_{G}(k)$ back inside the tolerable range. Obviously, this would not be satisfactory performance on power quality in the grid. 
When the system is in the emergency conditions above, the condition expressed in (16) or (19) is satisfied by definition, and so the central controller will ignore Steps B.4(b) and B.5 below, moving instead directly to Step B.6 for the next execution (see Fig. 8). In contrast, when neither (16) nor (19) is satisfied, the central controller will proceed with normal non-emergency operation, moving to Step B.4(b) below.

\section{b. Control in the transient state}

The individual reference value of active power for each PV agent can be determined as in (21), which is a modified version of a relation from [3],[4].

$$
\beta_{\text {Agent }, j}^{*}(k)=\left(1-\mu_{L, j}\right) \times\left[\frac{\beta_{\mathrm{Farm}, \Sigma}^{*}(k)}{\beta_{\mathrm{Farm}, \Sigma}^{\mathrm{Pre}, \mathrm{MrS}}(k)}\right] \times \beta_{\text {Agent }, j}^{\mathrm{Pre,Max}, \mathrm{TrS}}(k)
$$

The value is then clamped by (22), where $\beta_{\text {Agent, } j}^{\text {Pre,Max, } T r S}(k)$ is computed by (13).

$$
0 \leq \beta_{\text {Agent }, j}^{*}(k) \leq \beta_{\text {Agent }, j}^{\text {Pre,Max }, T r S}(k)
$$

From here, the central controller will skip Step B.5 and move directly to Step B.6.

\section{Step B.5: Control at the steady state}

$$
\beta_{B a t, j}^{\mathrm{Pre}, \mathrm{StS}}(k)=\left[S O C_{B a t, j}^{\mathrm{Meas}}(k)-S O C_{B a t}^{*, \mathrm{StS}}\right] \times \beta_{B a t, j}^{\mathrm{Rated}}
$$

At the steady state, $S O C_{B a t}^{*, S t S}=50 \%$ is the desired idle SOC for the battery banks.

The predicted total power capacities of a local PV agent $\beta_{\text {Agent }, j}^{\text {Pre,StS }}(k)$ and of PV farm $\beta_{\text {Farm }, \Sigma}^{\text {Pre,StS }}(k)$ at the steady state are given by (24) and (25), respectively.

$$
\begin{gathered}
\beta_{\text {Agent }, j}^{\text {Pre, } \mathrm{StS}}(k)=\beta_{P V \text { only }, j}^{\text {Pre }}(k)+\beta_{\text {Bat }, j}^{\text {Pre, }, \mathrm{StS}}(k) \\
\beta_{\mathrm{Farm}, \Sigma}^{\mathrm{Pre}, \mathrm{StS}}(k)=\sum_{j=1}^{N} \beta_{\text {Agent }, j}^{\mathrm{Pre}, \mathrm{StS}}(k)
\end{gathered}
$$

With the reference value of total power $\beta_{\text {Farm }, \Sigma}^{*}(k)$, which was already determined in (11) and (15), the individual reference value of active power for each local solar agent at the steady state can be calculated by (26).

$$
\beta_{\text {Agent }, j}^{*}(k)=\left(1-\mu_{L, j}\right) \times\left[\frac{\beta_{\text {Farm }, \Sigma}^{*}(k)}{\beta_{\text {Fre, } \mathrm{PtS}, \Sigma}^{\mathrm{Par}}(k)}\right] \times \beta_{\text {Agent }, j}^{\mathrm{PreStS}}(k)
$$

Here, the result is clamped by $0 \leq \beta_{\text {Agent }, j}^{*}(k) \leq$ $\beta_{P V \text { only,j }}^{\text {Pre,Max,TrS }}(k)$, shown as (22) above. Fig. 8).

The central controller move from here to Step B.6 (see
Step B.6: As the final step in the process, the individual reference value $\beta_{\text {Agent }, j}^{*}(k)$ computed above and the flag $S t S_{\text {Flag }}(k)$ to indicate the steady state will be sent from the central controller to the $j$ th local controller at each PV power agent for use in control.

\subsection{Determining the operations for four PV arrays and the battery bank in each local controller}

The design steps here were previously described in [14]. In this paper, Fig. 11 describes the flowchart of the steps, with some newly added detailed explanation in Steps C.1 and C.2. As presented in Fig. 9, the flag value $\operatorname{StS}_{\text {Flag }}(k)=1$ in Step C.3 is now used as the mandatory condition in regulating the SOC of the battery banks in the local PV agents at the steady state.

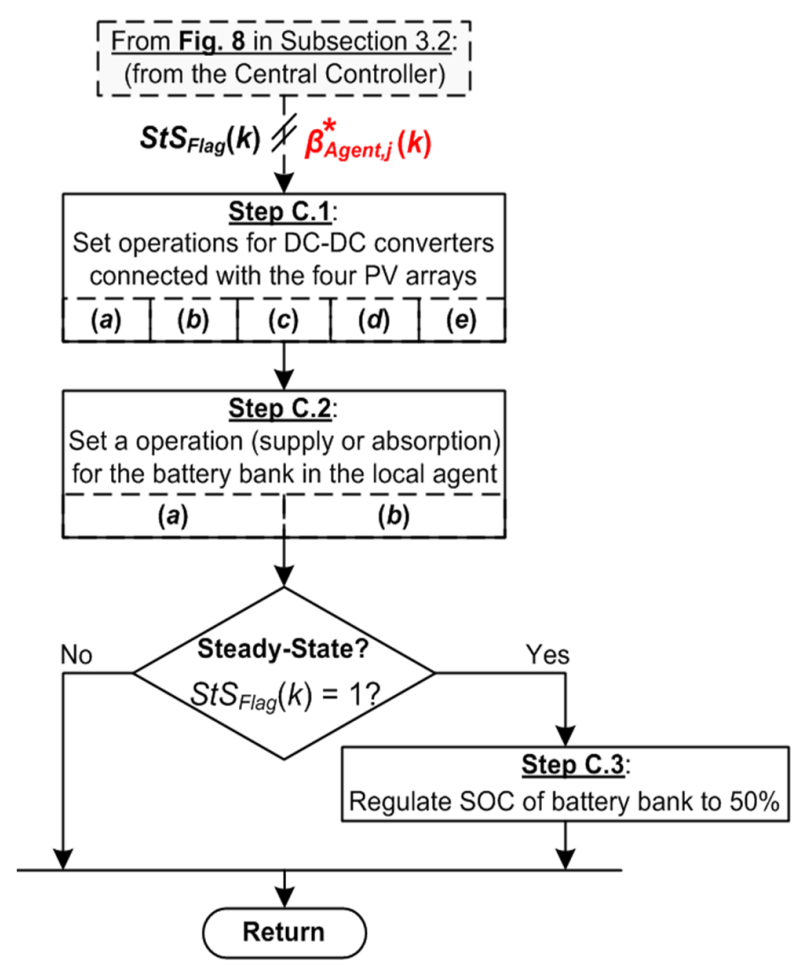

Fig. 11. Flowchart of the three design steps of Subsection 3.3.

Step C.1: A ratio value of power $\delta_{A g e n t, j}(k)$ as defined in (27) will be suitable for operations in the four DC-DC converters.

$$
\delta_{\text {Agent }, j}(k)=\left[\frac{\beta_{\text {Agent }, j}^{*}(k)}{\beta_{P V \text { Ponly }, j}^{\text {Pre }}(k)}\right] \times 100 \%
$$

In this research, the four PV arrays of each local agent are assumed to be within an area small enough that the predicted maximum power of each solar array can be taken as equal. In each local controller, according to the computed value of $\delta_{j}(k)$, one of five operation cases $(\boldsymbol{a}, \boldsymbol{b}, \boldsymbol{c}, \boldsymbol{d}$, and $\boldsymbol{e})$ will be set in each of the four DC-DC converters installed 
with the four PV arrays. It is notable that the particular operation mode for the battery bank has not yet been set in this step (which only predicts the operation of the battery bank). The operating mode (supply or absorb) of the battery bank is actually set in Step C.2. The cases are as follows.

a. If $\delta_{A g e n t, j}(k) \geq 100 \%$, all four DC-DC converters for the PV arrays will be set to operate in MPPT mode, and the battery bank is predicted to supply power.

b. If $75 \% \leq \delta_{\text {Agent }, j}(k)<100 \%$, the following two sub-cases are considered.

- If $S O C_{B a t, j}^{\mathrm{Meas}}(k)<S O C_{B a t}^{\mathrm{Max}}=80 \%$, all four DC-DC converters for the PV arrays will be controlled to operate in MPPT mode; meanwhile, the battery bank is predicted to absorb power.

- If $S O C_{B a t, j}^{\mathrm{Meas}}(k) \geq S O C_{B a t}^{\mathrm{Max}}$, the DC-DC converters for the first three PV arrays will be controlled to operate in MPPT mode; meanwhile, the DC-DC converter for the fourth PV array will be deactivated, and the battery bank is predicted to supply power.

c. If $50 \% \leq \delta_{\text {Agent }, j}(k)<75 \%$, the following two sub-cases are considered.

- If $S O C_{B a t, j}^{\mathrm{Meas}}(k)<S O C_{B a t}^{\mathrm{Max}}$, the DC-DC converters for the first three PV arrays will be controlled to operate in MPPT mode; meanwhile, the DC-DC converter for the fourth PV array will be deactivated, and the battery bank is predicted to would absorb power.

- If $S O C_{B a t, j}^{\mathrm{Meas}}(k) \geq S O C_{B a t}^{\mathrm{Max}}$, the DC-DC converters for the first and second $\mathrm{PV}$ arrays will be controlled to operate in MPPT mode; meanwhile, the DC-DC converters for the third and fourth PV arrays will be deactivated, and the battery bank is predicted to supply power.

d. If $25 \% \leq \delta_{\text {Agent }, j}(k)<50 \%$, the following two sub-cases are considered.

- If $S O C_{B a t, j}^{\mathrm{Meas}}(k)<S O C_{B a t}^{\mathrm{Max}}$, the DC-DC converters for the first and second $\mathrm{PV}$ arrays will be controlled to operate in MPPT mode; meanwhile, the DC-DC converters for the third and fourth PV arrays will be deactivated, and the battery bank is predicted to absorb power.

- If $S O C_{B a t, j}^{\mathrm{Meas}}(k) \geq S O C_{B a t}^{\mathrm{Max}}$, the DC-DC converter for the first PV array will be controlled to operate in MPPT mode; meanwhile, the DC-DC converters for the other PV arrays will be deactivated, and the battery bank is predicted to supply power.

e. In all other cases (that is, $0 \% \leq \delta_{\text {Agent }, j}(k)<25 \%$ ), the following two sub-cases are considered.

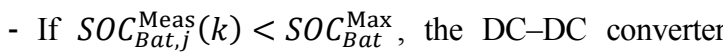
for the first PV array will be controlled to operate in MPPT mode; meanwhile, the DC-DC converters for the other PV arrays will be deactivated, and the battery bank is predicted to absorb power.

- If $S O C_{B a t, j}^{\mathrm{Meas}}(k) \geq S O C_{B a t}^{\mathrm{Max}}$, All DC-DC converters will be deactivated, and the battery bank is predicted to supply power.

Step C.2: Compute the amount of active power to be absorbed by or supplied from the battery bank, and set the operation mode (absorb or supply) for the battery bank at each local solar agent. The algorithm is as follows. First, the actual total power of all four PV arrays $\beta_{P V \text { only,j }}^{\text {Meas }}(k)$ in the local agent is measured by sensor for the current step; next, a difference value $\beta_{\text {Agent,j }}^{\text {Diff }}(k)$ is computed by (28); last, either case $\boldsymbol{a}$ or case $\boldsymbol{b}$ is chosen according to the conditions below.

$$
\beta_{\text {Agent }, j}^{\text {Diff }}(k)=\beta_{\text {Agent }, j}^{*}(k)-\beta_{P V \text { only }, j}^{\text {Meas }}(k)
$$

a. If $\beta_{\text {Agent }, j}^{\text {Diff }}(k)<0$, which implies $\beta_{A g e n t, j}^{*}(k)<$ $\beta_{\text {PVonly,j }}^{\text {Meas }}(k)$, the following two sub-cases are considered.

- If $S O C_{B a t, j}^{\mathrm{Meas}}(k)<S O C_{B a t}^{\mathrm{Max}}=80 \%$, the battery bank can absorb extra power; therefore, it will be controlled to absorb power according to (29) for the reference value for battery bank $\beta_{B a t, j}^{*}(k)$.

$$
\beta_{\text {Bat }, j}^{*}(k)=\beta_{\text {Agent }, j}^{\text {Diff }}(k)<0
$$

- If $\beta_{\text {Agent }, j}^{\text {Diff }}(k)<\beta_{B a t, j}^{\text {Pre,Absorb }}(k)$, then (30) is used instead.

$$
\beta_{\text {Bat }, j}^{*}(k)=\beta_{\text {Bat }, j}^{\text {Pre,Absorb }}(k)
$$

- Otherwise, (i.e., $S O C_{B a t, j}^{\mathrm{Meas}}(k) \geq S O C_{B a t}^{\mathrm{Max}}$ ), the battery bank cannot absorb extra power. So, in this case, the battery bank will be controlled to operate in neutral mode: $\beta_{B a t, j}^{*}(k)=0$.

b. If $\beta_{\text {Agent }, j}^{\text {Diff }}(k) \geq 0$, which implies $\beta_{\text {Agent }, j}^{*}(k) \geq$ $\beta_{P V O n l y, j}^{\text {Meas }}(k)$, the following two sub-cases are considered.

- If $S O C_{B a t, j}^{\text {Meas }}(k) \leq S O C_{B a t}^{\mathrm{Min}}=20 \%$, the battery bank cannot supply any more power. Thus, in this condition, the battery bank will be controlled to operate in neutral mode: $\beta_{B a t, j}^{*}(k)=0$.

- Otherwise (i.e., $S O C_{B a t, j}^{\mathrm{Meas}}(k)>S O C_{B a t}^{\mathrm{Min}}$ ), the battery bank will be controlled to supply power.

$$
\beta_{\text {Bat }, j}^{*}(k)=\beta_{\text {Agent }, j}^{\text {Diff }}(k) \geq 0
$$

- $\quad$ Note that if $\beta_{\text {Agent, } j}^{\text {Diff }}(k)>\beta_{B a t, j}^{\text {Pre,Supply,TrS }}(k)$, then (32) is used instead. 


$$
\beta_{\text {Bat }, j}^{*}(k)=\beta_{\text {Bat }, j}^{\text {Pre,Supply,TrS }}(k)
$$

Step C.3: When the power system is at the steady state, the SOC of the battery bank is adjusted toward reaching the desired idle ratio value of $S O C_{B a t}^{*, \text { ttS }}=50 \%$. The goal of this task is to guarantee that the battery bank can absorb or supply power as needed at the next operation time. The detailed procedure to regulate the SOC of the battery bank is as follows.

- If the power system is at the steady state, this means that $S t S_{\text {Flag }}(k)=1$, and the following sub-cases are considered.

- If $S O C_{B a t, j}^{\mathrm{Meas}}(k)<S O C_{B a t}^{*, \mathrm{StS}}=50 \%$, the bidirectional DC-DC battery charger at the jth local agent will be controlled to absorb power from the PV arrays to boost SOC of the battery bank toward reaching the desired idle ratio value of $50 \%$.

- If $S O C_{B a t, j}^{\mathrm{Meas}}(k)>S O C_{B a t}^{*, \mathrm{StS}}$, the DC-DC battery charger at the $j$ th local agent will be controlled to inject power from the battery bank into the grid via the $\mathrm{DC}-\mathrm{AC}$ inverter in order to reduce the SOC of the battery bank toward the desired idle ratio value of $50 \%$.

- Otherwise, (i.e., $S O C_{B a t, j}^{\mathrm{Meas}}(k)=S O C_{B a t}^{*, \mathrm{StS}}$ ), the battery bank at the $j$ th local PV agent will continue to operate in neutral mode.

\subsection{Design of the newly proposed FLC scheme}

First, the magnitude of difference between the previous reference signal $\beta_{\text {Farm }, \Sigma}^{*}(k-1)$ and the previous actual total power $\beta_{\text {Farm }, \Sigma}^{\text {Meas }}(k-1)$ supplied to the grid from a PV farm is calculated by (33).

$$
\beta_{\text {Farm }}^{\text {Diff }}(k-1)=\left|\beta_{\text {Farm }, \Sigma}^{*}(k-1)-\beta_{\text {Farm }, \Sigma}^{\text {Meas }}(k-1)\right|
$$

The ratio value in percent $\delta_{\text {Farm }}^{\text {Diff }}(k-1)$ is given by (34).

$$
\delta_{\text {Farm }}^{\text {Diff }}(k-1)=\left\{\begin{array}{l}
\frac{\beta_{\text {Farm }}^{\text {Diff }}(k-1)}{\beta_{\text {Farm }, \Sigma}^{*}(k-1)} \times 100 \%, \text { if } \beta_{\text {Farm }, \Sigma}^{*}(k-1)>\varepsilon \\
\frac{\beta_{\text {Farm }}^{\text {Diff }}(k-1)}{\varepsilon} \times 100 \%, \text { if } 0 \leq \beta_{\text {Farm }, \Sigma}^{*}(k-1) \leq \varepsilon
\end{array}\right.
$$

In (34), $\varepsilon$ is a positive value that is very small relative to 1 (p.u.); in this study, $\varepsilon$ is set as 0.001 (p.u.) $<<1$ (p.u.).

After this, the grid frequency deviation, shown in Fig. 2 as $E f_{G}(k)$, its stepwise change $\Delta E f_{G}(k)$, the difference ratio value $\delta_{\text {Farm }}^{\text {Diff }}(k-1)$ from (34), and the steady-state indication flag $S t S_{\text {Flag }}(k)$ from Step B.3 are used as inputs for the proposed automatic-tuning FLC scheme as illustrated in Fig. 12.

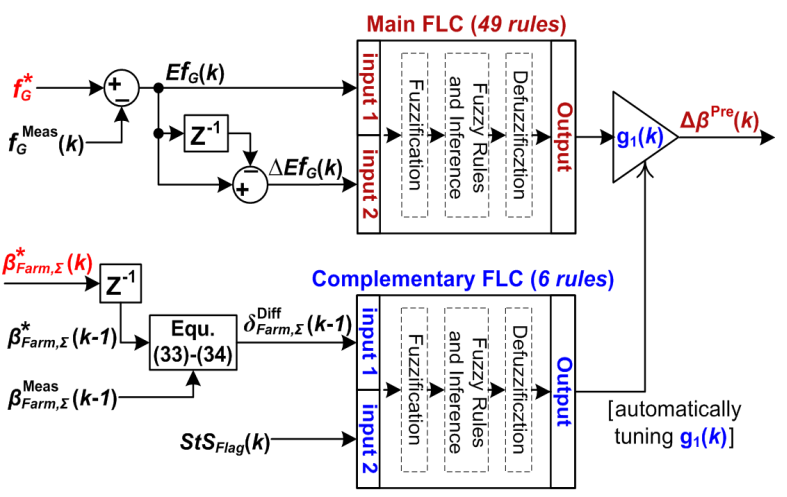

Fig. 12. Structure of the FLC scheme shown in Fig. 4(a).

\subsubsection{The main 49-rule FLC}

This 49-rule FLC is enhanced substantially from the version presented in [14]. Some useful improvements have been made, and the design parameters have been optimized.

As illustrated in the upper block of Fig. 12, the main 49-rule FLC uses the grid frequency error $E f_{G}(k)$, shown as the frequency deviation in Fig. 2(a), and its step variation $\Delta E f_{G}(k)$ as inputs to calculate the adjustment value $\Delta \beta^{\text {Pre }}(k)$. The calculated value of $\Delta \beta^{\text {Pre }}(k)$ will be used for online updating of the reference value for total active power $\beta_{\text {Farm }, \Sigma}^{*}(k)$ as given by (11). See Subsection 3.2 for details. The features of the main FLC are described here.

Two inputs: The inputs have seven linguistic variables and values in the range -0.25 to $0.25(\mathrm{~Hz})$. The limiting values of $\pm 0.25(\mathrm{~Hz})$ where chosen as equal to $125 \%$ of the design range of the range $\pm 0.2(\mathrm{~Hz})$ applied to grid frequency deviation. The designed value range of -0.25 to 0.25 can be chosen relatively independently of the nominal total power capacity of the PV farm.

$$
\begin{aligned}
E f_{G}(k)=\{ & \text { Negative Large, Negative Medium, Negative } \\
& \text { Small, Zero, Positive Small, Positive Medium, } \\
& \text { Positive Large }\} \\
=\{ & \text { NL, NM, NS, ZR, PS, PM, PL }\} \\
\Delta E f_{G}(k)= & E f_{G}(k)-E f_{G}(k-1) \\
= & \{\text { Negative Large, Negative Medium, Nega- } \\
& \text { tive Small, Zero, Positive Small, Positive } \\
& \text { Medium, Positive Large }\} \\
= & \{\text { NL, NM, NS, ZR, PS, PM, PL }\}
\end{aligned}
$$

The output: The output has nine linguistic variables and a value in the range -0.64 to 0.64 (p.u.). This limit for $\Delta \beta^{\text {Pre }}(k)$ is determined from the following: $\pm S O C_{B a t}^{\text {Max }} \times$ $\beta_{\text {Bat }, j}^{\text {Rated }}= \pm 80 \% \times 0.8$ (p.u.) $= \pm 0.64$ (p.u.). Thus, the designed value range of -0.64 to 0.64 for $\Delta \beta^{\text {Pre }}(k)$ depends primarily on the power capacity of the battery bank installed at each local agent, and is independent of the number of agents in the PV farm.

$\Delta \beta^{\text {Pre }}(k)=\{$ Negative Extreme, Negative Large, Negative Medium, Negative Small, Zero, Positive Small, Positive Medium, Positive Large, Positive Extreme\} 
$=\{\mathrm{NE}, \mathrm{NL}, \mathrm{NM}, \mathrm{NS}, \mathrm{ZR}, \mathrm{PS}, \mathrm{PM}, \mathrm{PL}, \mathrm{PE}\}$

Membership functions:

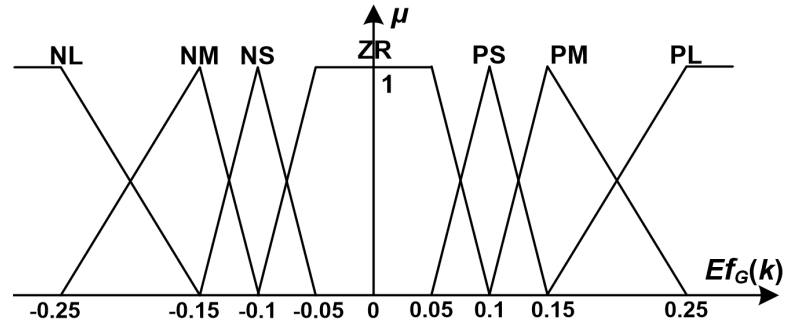

(a) Membership functions for the first input

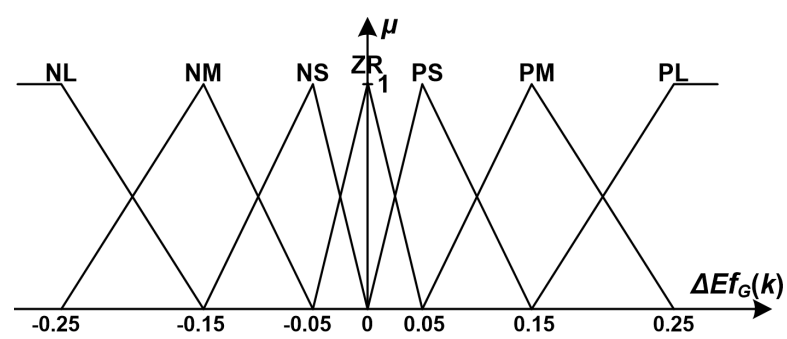

(b) Membership functions for the second input

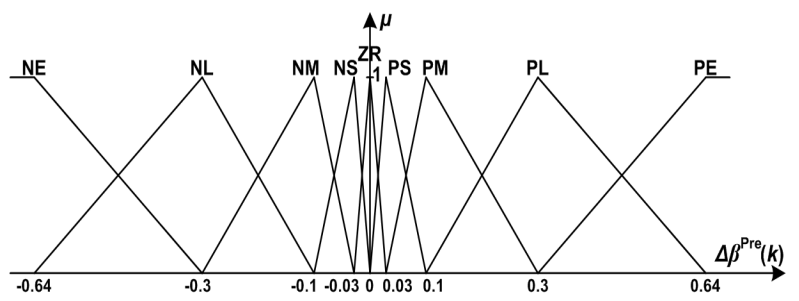

(c) Membership functions for the output

Fig. 13. Membership functions for the main 49-rule FLC.

The membership functions for the two inputs and output of the main 49-rule FLC are shown in Figs. 13 panels (a), (b), and (c), respectively. The particular shapes of membership functions were adjusted manually by the authors to reflect the following priorities. First, if the grid frequency deviation $E f_{G}(k)$ is far outside the acceptable standard range of \pm 0.2 $(\mathrm{Hz})$, then the adjustment value of power $\Delta \beta^{\text {Pre }}(k)$ must be quite large to swiftly regulate $E f_{G}(k)$ into the tolerable range of $\pm 0.2(\mathrm{~Hz})$. After that, if the power system is at the steady state, then $\Delta \beta^{\mathrm{Pre}}(k)$ should be chosen to be small to help dampen fluctuations in $E f_{G}(k)$.

Moreover, as presented in Section 1 and Fig. 9, the very small range of $\pm 0.05(\mathrm{~Hz})$ is chosen as the limit of the grid frequency deviation at the steady state. Therefore, in Fig. 13(a), the range of $\pm 0.05(\mathrm{~Hz})$ is used to design the main area of linguistic variable $\mathrm{ZR}$ of the first input $E f_{G}(k)$, and it is the full range of the variable $\mathrm{ZR}$ of the second $\Delta E f_{G}(k)$ as given in Fig. 13(b). Because the two inputs of the 49-rule FLC are designed with both negative and positive values, the membership functions for the ZR variables of these inputs should be symmetrical around the value of 0 . In a similar way, the linguistic variable ZR of the output of the FLC should have a very small range compared with the limiting range of \pm 0.64 . Therefore, as shown in Fig. 13(c), the range for the variable ZR of the output is chosen as \pm 0.03 , which is approximately equal to $4.7 \%(<5 \%)$ of the limiting interval of \pm 0.64 .

Table 2. Fuzzy association rules for the main 49-rule FLC.

\begin{tabular}{|c|c|c|c|c|c|c|c|c|}
\hline \multirow{2}{*}{\multicolumn{2}{|c|}{$\Delta \beta^{\operatorname{Pre}}(k)$}} & \multicolumn{7}{|c|}{$\boldsymbol{E} \boldsymbol{f}_{G}(\boldsymbol{k})$} \\
\hline & & NL & NM & NS & ZR & PS & PM & PL \\
\hline \multirow{7}{*}{$\Delta E f_{G}(k)$} & NL & $\mathrm{PE}$ & $\mathrm{PE}$ & $\mathrm{PE}$ & PE & PL & PS & NS \\
\hline & $\mathrm{NM}$ & $\mathrm{PE}$ & $\mathrm{PE}$ & $\mathrm{PE}$ & PM & PS & ZR & $\mathrm{NM}$ \\
\hline & NS & $\mathrm{PE}$ & $\mathrm{PE}$ & PM & PS & ZR & NS & $\mathrm{NL}$ \\
\hline & ZR & $\mathrm{PE}$ & PL & PS & ZR & NS & NL & $\mathrm{NE}$ \\
\hline & PS & PL & PS & ZR & NS & NM & $\mathrm{NE}$ & $\mathrm{NE}$ \\
\hline & PM & PM & ZR & NS & $\mathrm{NM}$ & $\mathrm{NE}$ & $\mathrm{NE}$ & $\mathrm{NE}$ \\
\hline & PL & PS & NS & $\mathrm{NM}$ & $\mathrm{NE}$ & $\mathrm{NE}$ & $\mathrm{NE}$ & $\mathrm{NE}$ \\
\hline
\end{tabular}

Interpretation for the fuzzy association rules in Table 2:

As shown in Table 2, a sample rule expressed as "if $E f_{G}(k)$ is NL and $\Delta E f_{G}(k)$ is NL then $\Delta \beta^{\text {Pre }}(k)$ is PE" is employed for analysis. Because the frequency error $E f_{G}(k)=\left[f_{G}^{*}-f_{G}^{\text {Meas }}(k)\right]$ is NL (negative large), the grid frequency $f_{G}^{\text {Meas }}(k)$ is much larger than its desired value of $f_{G}^{*}=60(\mathrm{~Hz})$; meanwhile, the change of frequency error $\Delta E f_{G}(k)$ is also NL (negative large). Because of this, the frequency error $E f_{G}(k)$ may continue to decrease rapidly; this indicates a tendency for the grid frequency $f_{G}^{\text {Meas }}(k)$ to become markedly larger than $f_{G}^{*}=60(\mathrm{~Hz})$. To resolve this issue, the reference value of total power $\beta_{\text {Farm }, \Sigma}^{*}(k)$ is added with a very large positive quantity. To do so, $\beta_{F a r m, \Sigma}^{*}(k)$ must be significantly large relative to the actual total power previously injected into the grid, $\beta_{F a r m, \Sigma}^{\text {Meas }}(k-$ 1 ), so that the grid frequency $f_{G}^{\text {Meas }}(k)$ can be sharply and swiftly reduced. The final goal is to promptly raise the deviation $E f_{G}(k)$ to zero. For this, the $k$ th adjustment value $\Delta \beta^{\text {Pre }}(k)$ should be chosen as PE (positive extreme).

Otherwise, in another case, the error value $E f_{G}(k)$ is still NL (negative large) as in the above situation, but the change of error $\Delta E f_{G}(k)$ is now changed to PL (positive large). This means that the grid frequency $f_{G}^{\text {Meas }}(k)$ will tend to decrease automatically toward $f_{G}^{*}=60(\mathrm{~Hz})$. To boost the response speed and minimize overshooting in the transient state, $\beta_{F a r m, \Sigma}^{*}(k)$ should be increased slightly. Thus, $k$ th adjustable value $\Delta \beta^{\text {Pre }}(k)$ could be set as PS (positive small). Broadly, a similar series of deductions can be used to interpret the other fuzzy rules presented in Table 2. In addition, based on results obtained from simulations, several rules can be modified and optimized manually.

\subsubsection{The complementary 6-rule FLC}

The simple 6-rule FLC is newly developed to adjust the output scaling factor $g_{1}(k)$ of the main 49-rule FLC automatically and suitably, as shown in Fig. 12. This means that the output factor $g_{1}(k)$ can be changed online, instead being fixed (in [14], it was regarded as fixed). The goal of 
this change is to help force the difference ratio value $\delta_{\text {Farm }}^{\text {Diff }}(k-1)$ from $(34)$ to become a tolerable value in the transient state and nearly zero at the steady state. Of course, this also helps to improve adaptability of the proposed FLC scheme under conditions of parametric uncertainty. Moreover, this complementary FLC can assist with supervising and regulating the difference between the previous reference signal $\beta_{F a r m, \Sigma}^{*}(k-1)$ and the previous actual total power $\beta_{\text {Farm }, \Sigma}^{\text {Meas }}(k-1)$ supplied from the PV farm in real time. The features of the complementary FLC are described here.

\section{Two inputs:}

- The first input has three linguistic variables and a value in the range $0-8 \%$ as shown in Fig. 14(a). The linguistic variable SM (small) is set to be $0 \%$ as a very small value for the error $\delta_{\text {Farm }}^{\text {Diff }}(k-1)$. In real control systems, acceptable values for the error at the steady state are normally in the range of $1-5 \%$. Hence, in this study, the linguistic variable ME (medium) of the error is chosen as the average value of this range as $(1 \%+$ $5 \%) / 2=3 \%$. However, the value for $\delta_{\text {Farm }}^{\text {Diff }}(k-1)$ in the transient state (which is fairly near the steady state) is often expected to be in the range $6-10 \%$, so the linguistic variable LA (large) of the $\delta_{\text {Farm }}^{\text {Diff }}(k-1)$ is chosen as the average value of this range, as $(6 \%+10 \%) / 2=8 \%$.

$$
\begin{aligned}
\delta_{\text {Farm }}^{\text {Diff }}(k-1) & =\{\text { Small, Medium }, \text { Large }\} \\
& =\{\text { SM, ME }, \text { LA }\}
\end{aligned}
$$

- As already illustrated in Fig. 9, the indication flag $\operatorname{StS}_{\text {Flag }}(k)$ is either 0 or 1 . Thus, the second input of the complementary FLC is designed with two linguistic singleton variables and has a value in the range $[0,1]$ as given in Fig. 14(b).

$$
\begin{aligned}
\text { StS } S_{\text {Flag }}(k) & =\{\text { Transient State, Steady State }\} \\
& =\{\mathrm{TrS}, \mathrm{StS}\}
\end{aligned}
$$

The output: The output has four linguistic variables and a value in the range of $[0,1.2]$; as well, $0 \leq g_{1}(k) \leq 1.2$.

$$
\begin{aligned}
g_{1}(k) & =\{\text { Very Small, Small, Medium, Large }\} \\
& =\{\text { VS, SM, ME, LA }\}
\end{aligned}
$$

Normally, the output scaling factor of a traditional FLC is set as a fixed value of 1 . From test results obtained in numerical simulations of this study, the suitable value chosen for the linguistic variable ME (medium) of $g_{1}(k)$ is 0.9 ; and the value set for the linguistic variable SM (small) is 0.3 , which can be considered as an acceptably small value to reduce large fluctuation of $g_{1}(k)$ in the nearly steady state. Meanwhile, the value for the linguistic variable VS (very small) can be assigned as 0 to help eliminate thoroughly oscillation of $g_{1}(k)$ at the steady state. Lastly, the value for the linguistic variable LA (large) often must be larger than 1; and in this study, it is chosen as $0+0.3+0.9=1.2$, which is the total of the three values utilized for the prior variables. Besides, the output value of the 6-rule FLC, $g_{1}(k)$, is automatically tuned inside the value range of $[0,1.2]$. Hence, its initial value $g_{1}(k=0)$ should be optionally chosen as a small value in the interval of $[0,1.2]$. In this study, for simplicity, this value can be set as $g_{1}(k=0)=0$.

Membership functions for the two inputs and the output of the complementary 6-rule FLC are given in Fig. 14 panels (a), (b), and (c), respectively. As shown in Figs. 14(a) and (c), the membership functions are asymmetrical triangular types; the membership functions in Fig. 14(b) are singleton types. Moreover, note that all the inputs and the output of this 6-rule FLC have non-negative value ranges to simplify the structure, the ultimate goal of which is to help reduce the overall computational time of the proposed strategy.

\section{Membership functions:}

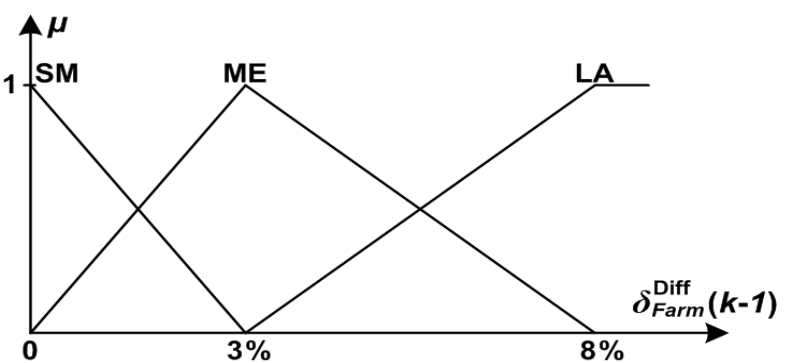

(a) Membership functions for the first input

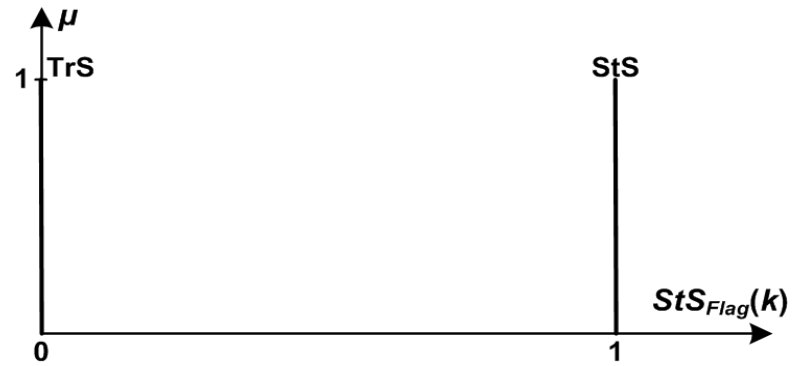

(b) Membership functions for the second input

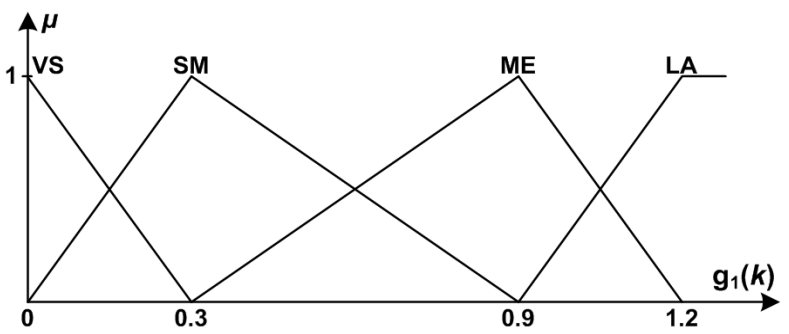

(c) Membership functions for the output

Fig. 14. Membership functions for the 6-rule FLC.

Table 3. Fuzzy association rules for the 6-rule FLC.

\begin{tabular}{|c|c|c|c|c|}
\hline \multirow{2}{*}{$\boldsymbol{g}_{\mathbf{1}}(\boldsymbol{k})$} & \multicolumn{3}{c|}{$\boldsymbol{\delta}_{\text {Farm }}^{\text {Diff }}(\boldsymbol{k}-\mathbf{1})$} \\
\cline { 3 - 5 } & $\mathrm{SM}$ & $\mathrm{ME}$ & $\mathrm{LA}$ \\
\hline \multirow{2}{*}{$\boldsymbol{S t S}_{\text {Flag }}(\boldsymbol{k})$} & $\mathrm{TrS}$ & $\mathrm{ME}$ & $\mathrm{ME}$ & $\mathrm{LA}$ \\
\cline { 2 - 5 } & $\mathrm{StS}$ & $\mathrm{VS}$ & $\mathrm{VS}$ & $\mathrm{SM}$ \\
\hline
\end{tabular}


Interpretation for the fuzzy association rules in Table 3:

As described in the above table, a sample rule expressed as "if $\delta_{\text {Farm }}^{\text {Diff }}(k-1)$ is LA and $S t S_{\text {Flag }}(k)$ is $\operatorname{TrS}$ then $g_{1}(k)$ is LA" is used for analysis. In this case, because the difference ratio value $\delta_{\text {Farm }}^{\text {Diff }}(k-1)$ is LA (large), the actual total power supplied from the PV farm has not yet achieved its reference value; meanwhile, the indication flag $S t S_{\text {Flag }}(k)$ is $\operatorname{TrS}$ (transient state). It can be predicted that the grid frequency deviation $E f_{G}(k)$ is outside the range of $\pm 0.05(\mathrm{~Hz})$ at the current step and that the power system is in the settling time of the transient state. Therefore, the output scaling factor of the main 49-rule FLC $g_{1}(k)$ should be large to help force the frequency error $E f_{G}(k)$ to move speedily into the range $\pm 0.05(\mathrm{~Hz})$. The value for $g_{1}(k)$ should therefore be chosen as LA (large). In a different case, if $\operatorname{StS}_{\text {Flag }}(k)$ is still $\mathrm{TrS}$, as before, but the difference ratio value $\delta_{\text {Farm }}^{\text {Diff }}(k-1)$ is SM (small), then the power system seems to be nearly at the steady state. Hence, the value for $g_{1}(k)$ should be reduced from the LA (large) given for the above case to a new value of ME (medium) to eliminate, in advance, the overshoot for $\delta_{\text {Farm }}^{\text {Diff }}(k-1)$.

In contrast, with the lowest row of Table 3, if the indication flag $S t S_{\text {Flag }}(k)$ is StS (steady state), this means that the grid frequency deviation $E f_{G}(k)$ is currently in the small range $\pm 0.05(\mathrm{~Hz})$. According to the priority of maintaining the grid frequency, the values for $g_{1}(k)$ at the steady state need to be chosen small relative to the values for the transient state (given in the upper row). In general, when the power system is in the transient state, where $S t S_{\text {Flag }}(k)$ is $\operatorname{TrS}$, the highest priority is to substantially boost the speed of adjusting the total power injected into the grid in order to damp quickly the grid frequency deviation $E f_{G}(k)$ into range $\pm 0.05(\mathrm{~Hz})$; for this, $g_{1}(k)$ could be set as a medium or large value. In other case, when the system operates at the steady state, where $S t S_{\text {Flag }}(k)$ is $\mathrm{StS}, g_{1}(k)$ should be set as small or very small to make only slight and slow adjustments to the total power delivered to the grid with the aim of suppressing steady-state fluctuation of $E f_{G}(k)$. As well, this design technique for the 6-rule FLC also considers the difference ratio value $\delta_{\text {Farm }}^{\text {Diff }}(k-1)$ to more flexibly determine a suitable value for $g_{1}(k)$ and can help regulate $\beta_{\text {Farm }}^{\text {Diff }}(k-1)$ of (33) so that it lies in an acceptably small range.

\subsubsection{Fuzzy inference system and defuzzification}

In the two FLCs introduced in Fig. 12, the Max-Min composition operation and the Centroid (CoA: center of area) methods of [27],[28] are used for fuzzy inference and defuzzification, respectively.

\section{Simulation Results}

In this paper, three simulation cases are described. These were performed in MATLAB [29]-[34]. The authors in [29] presented a sample simulation of an electricity grid in MATLAB, and the studies in [30]-[34] successfully applied this software in simulating artificial intelligence methods. The first case considered one large-scale PV farm, with sudden large changes in solar irradiance and AC system load (Subsection 4.1.1). The second case considered one large-scale PV farm with unexpected changes in air temperature and two main parameters of AGC (Subsection 4.1.2). In fact, these first two test cases (see Table 4 for parameter settings) clearly show the operating principles and efficacy of the proposed fuzzy-based strategy. The third and final case considered a four-area power system, in which four large-scale PV farms are connected and the regulation of tie-line power flows between the areas is implemented (Subsection 4.2).

Additionally, the conventional simple strategy, which is based on using full MPPT mode at the PV farm, will be considered as the reference strategy against the proposed strategy. In this conventional strategy based on full MPPT mode, the power produced by all PV arrays installed at the solar farm is injected into the grid, and all battery banks are controlled to operate in neutral mode.

\subsection{Simulations with one PV farm connected}

The configurations of the simulated PV farm and grid are given in Table 4 and are partially described in Step A.2 of Subsection 3.1. According to Step A.2, (7), (14), and (15), where $\beta_{P V \text { only }, j}^{\text {Rated }}=0.0167$ (p.u.), $\beta_{B a t, j}^{\text {Rated }}=0.0133$ (p.u.), and $N=3$, the peak total power possible from the PV farm, including all solar arrays and battery banks in the three local agents, can be estimated as $3 \times[0.0167$ p.u. $+(100 \%-$ $20 \%) \times 0.0133$ p.u.] $=0.082$ (p.u.). It is noted that 1 (p.u.) corresponds to the normalized grid power capacity $P_{\text {Grid }}^{\text {Capacity }}=P_{p . u}^{\text {Base }}=6(\mathrm{MW})$, where the nominal grid frequency is $60(\mathrm{~Hz})$.

Table 4. Parameters of the PV farm for Case 1 and Case 2.

\begin{tabular}{|c|}
\hline Parameter values of local PV agent in Fig. 1 and (1) \\
$n_{\text {Strings }}=10 ; n_{P V \text { panels }}=10 ; P_{\text {panel }}^{\text {Rated }}=250(\mathrm{~W})$ \\
\hline Number of local PV agents in the solar farm: $N=3$ \\
\hline Parameter values of the power system in Fig. 2(a) \\
$B=20 ; K i=0.4 ; R=0.1 ; T_{G}=0.2 ; T_{T}=0.5 ;$ \\
$M=3.5 ; \quad D=1 ; T_{B}=0.1$ \\
Grid power capacity: $P_{\text {Grid }}^{\text {Capacity }}=6(\mathrm{MW})$ \\
\hline Power system and control sampling times \\
$T_{S-\text { System }}=0.1(\mathrm{~s}) ; T_{S-\text { Control }}=0.05(\mathrm{~s})$ \\
\hline
\end{tabular}

\subsubsection{Case 1: Changes in solar radiation and AC load}

Table 5 shows environmental conditions in this case. Air temperature at all PV agents is fixed at $25\left({ }^{\circ} \mathrm{C}\right)$ during operation; the $\mathrm{AC}$ system loads and solar radiation levels at the three local PV agents are changed as follows. In the first period $t=[0 \mathrm{~s}, 20 \mathrm{~s}$ ), the nominal value is 0.082 (p.u.) for load and $1\left(\mathrm{~kW} / \mathrm{m}^{2}\right)$ for radiation level at all three local agents. In the second period $t=[20 \mathrm{~s}, 35 \mathrm{~s})$, the AC system load is decreased by $68 \%$ to become 0.0263 (p.u.); the solar radiation at the first local agent is kept at $1\left(\mathrm{~kW} / \mathrm{m}^{2}\right)$; the solar radiation at the second local agent is lowered to 0.6 $\left(\mathrm{kW} / \mathrm{m}^{2}\right)$; and the solar radiation at the third local agent is increased to $1.2\left(\mathrm{~kW} / \mathrm{m}^{2}\right)$. Finally, in the third operational period $t=[35 \mathrm{~s}, 50 \mathrm{~s}]$, the AC system load is increased to 
0.0566 (p.u.); the solar radiation at the second local PV agent rises to $1\left(\mathrm{~kW} / \mathrm{m}^{2}\right)$; and solar radiations at the first and third local PV agents become $0.85\left(\mathrm{~kW} / \mathrm{m}^{2}\right)$.

Table 5. Solar radiation and AC load for Case 1.

\begin{tabular}{|c|c|c|c|}
\hline \multirow[b]{2}{*}{$\begin{array}{l}\text { Three agents } \\
\text { in PV farm }\end{array}$} & \multicolumn{3}{|c|}{ Three operational time periods } \\
\hline & $\begin{array}{l}\text { 1st period } \\
t=[0,20) \mathrm{s}\end{array}$ & $\begin{array}{c}\text { 2nd period } \\
t=[20,35) \mathrm{s}\end{array}$ & $\begin{array}{c}\text { 3rd period } \\
t=[35,50] \mathrm{s}\end{array}$ \\
\hline 1st local agent & $1 \mathrm{~kW} / \mathrm{m}^{2}$ & $1 \mathrm{~kW} / \mathrm{m}^{2}$ & $0.85 \mathrm{~kW} / \mathrm{m}^{2}$ \\
\hline 2nd local agent & $1 \mathrm{~kW} / \mathrm{m}^{2}$ & $0.6 \mathrm{~kW} / \mathrm{m}^{2}$ & $1 \mathrm{~kW} / \mathrm{m}^{2}$ \\
\hline 3rd local agent & $1 \mathrm{~kW} / \mathrm{m}^{2}$ & $1.2 \mathrm{~kW} / \mathrm{m}^{2}$ & $0.85 \mathrm{~kW} / \mathrm{m}^{2}$ \\
\hline AC system load & 0.082 p.u. & 0.0263 p.u. & 0.0566 p.u. \\
\hline Temperatures & \multicolumn{3}{|c|}{ fixed at $25^{\circ} \mathrm{C}$} \\
\hline
\end{tabular}

Performances of the PV farm in this case are shown in Figs. 15 to 19. As seen in Fig. 15, the frequency deviation is much smaller when using the suggested coordinated strategy than when using the conventional (reference) strategy. The deviation is kept in the range $\pm 0.2(\mathrm{~Hz})$ in the transient state and in the range $\pm 0.05(\mathrm{~Hz})$ at the steady state. Under the conventional strategy, using full MPPT mode causes the threshold value of $\pm 0.2(\mathrm{~Hz})$ to be exceeded for periods [0.4 s, $1.3 \mathrm{~s}],[20.2 \mathrm{~s}, 21.5 \mathrm{~s}],[21.98 \mathrm{~s}, 22.86 \mathrm{~s}]$, and $[35.4 \mathrm{~s}, 36 \mathrm{~s}]$. The response speed to achieve the steady state under the suggested strategy is markedly faster than that under the conventional strategy. For example, in the first period, the proposed strategy takes about 3 seconds, from $t=0(\mathrm{~s})$ to $t=3(\mathrm{~s})$, to reach the steady state; in contrast, the conventional strategy takes about 13 seconds, from $t=0(\mathrm{~s})$ to $t=13(\mathrm{~s})$, to reach the steady state. At the beginning of the second period $t=20(\mathrm{~s})$, due to the sudden large reduction of $\mathrm{AC}$ load, the special control mode in (17) is activated to recover rapidly the grid frequency.

Furthermore, the actual total power delivered from the PV farm to the grid is controlled according to the reference value computed by the central controller as presented in Fig. 16. The power actually injected from each local PV agent is changed according to the three different operational conditions, as given in Fig. 17. As expressed in Fig. 18, the output factor $g_{1}(k)$ of the proposed FLC scheme (Fig. 12) is changed online to help properly tune the reference value. As shown in Fig. 19, the battery banks installed at three local PV agents are driven to supply power in three periods (roughly, [0 s, $1.6 \mathrm{~s}$ ], [23.4 s, $30.4 \mathrm{~s}$ ], and [35.1 s, $36.6 \mathrm{~s}]$ ) and to absorb power in three periods (roughly, [1.6 s, $7.5 \mathrm{~s}$, [20.1 s, $23.4 \mathrm{~s}]$, and [36.6 s, $42.2 \mathrm{~s}])$. In fact, by using the proposed control strategy, the appropriate operations for the battery banks at the agents were set, which plainly helped to reduce substantially the deviation in grid frequency, particularly when the AC system load and solar irradiance abruptly changed at $t=20(\mathrm{~s})$ and $t=35(\mathrm{~s})$.

\subsubsection{Case 2: Changes in air temperature and parameters of AGC for synchronous generator}

In this simulation case, the air temperature at each local PV agent is assumed to alter abruptly and noticeably (see Table 6 for values).
Table 6. Air temperatures and AGC parameters in Case 2.

\begin{tabular}{|c|c|c|c|}
\hline \multirow{2}{*}{$\begin{array}{c}\text { Three agents } \\
\text { in PV farm }\end{array}$} & \multicolumn{3}{|c|}{ Three operational time periods } \\
\cline { 2 - 4 } & $\begin{array}{c}1 \text { st period } \\
t=[0,20) \mathrm{s}\end{array}$ & $\begin{array}{c}\text { 2nd period } \\
t=[20,30) \mathrm{s}\end{array}$ & $\begin{array}{c}\text { 3rd period } \\
t=[30,50] \mathrm{s}\end{array}$ \\
\hline 1st local agent & $40^{\circ} \mathrm{C}$ & $20^{\circ} \mathrm{C}$ & $25^{\circ} \mathrm{C}$ \\
\hline 2nd local agent & $40^{\circ} \mathrm{C}$ & $25^{\circ} \mathrm{C}$ & $25^{\circ} \mathrm{C}$ \\
\hline 3rd local agent & $40^{\circ} \mathrm{C}$ & $15^{\circ} \mathrm{C}$ & $25^{\circ} \mathrm{C}$ \\
\hline $\begin{array}{c}\text { Two parameters } \\
\text { of AGC }\end{array}$ & $\begin{array}{c}B=20 ; R=0.1 \\
\text { (as shown in Table 4) }\end{array}$ & $\begin{array}{c}B=6.2 ; \\
R=0.323\end{array}$ \\
\hline Radiation, Load & fixed at $1 \mathrm{~kW} / \mathrm{m}^{2}$ and $0.082 \mathrm{p} . \mathrm{u}$. \\
\hline
\end{tabular}

The solar radiation levels at the local PV agents and the AC system load are fixed during operation at $1\left(\mathrm{~kW} / \mathrm{m}^{2}\right)$ and 0.082 (p.u.), respectively. The air temperatures at the local PV agents change as follows. In the first period $t=[0 \mathrm{~s}$, $20 \mathrm{~s})$, of the temperature is $40\left({ }^{\circ} \mathrm{C}\right)$ at all local agents. In the second period $t=[20 \mathrm{~s}, 30 \mathrm{~s})$, the air temperatures at the agents decrease to $20\left({ }^{\circ} \mathrm{C}\right), 25\left({ }^{\circ} \mathrm{C}\right)$, and $15\left({ }^{\circ} \mathrm{C}\right)$, for the first, second, and third agent, respectively. In the third period $t=[30 \mathrm{~s}, 50 \mathrm{~s}]$, the air temperature becomes $25\left({ }^{\circ} \mathrm{C}\right)$ at all agents; however, two parameters of the AGC, previously at $B=20$ and $R=0.1$, become $B=6.2$ and $R=0.323$. In fact, the new values of $B$ and $1 / R$ are reduced to $31 \%$ of the previous amounts.

Performances of the PV farm in this test are illustrated by Figs. 20 to 24. In Fig. 20, the grid frequency deviation under the proposed fuzzy-based strategy is markedly smaller than under the conventional strategy. In fact, the frequency is stabilized well to have the deviation in the range $\pm 0.2(\mathrm{~Hz})$ for the transient state and within $\pm 0.05(\mathrm{~Hz})$ at the steady state. In contrast, under the conventional strategy, the grid frequency deviation exceeds $\pm 0.2(\mathrm{~Hz})$ in three periods (roughly, [0.29 s, $1.3 \mathrm{~s}],[2.15 \mathrm{~s}, 2.6 \mathrm{~s}]$, and $[31.34 \mathrm{~s}, 33.1 \mathrm{~s}])$. The response speed for stabilizing the frequency is considerably better under the suggested strategy than under the conventional strategy.

Indeed, as illustrated in Fig. 21, for the proposed strategy, the total power supplied to the grid from the PV solar farm is controlled nearly exactly to the reference value defined in advance by the central controller. As seen in Fig. 22 , the individual power delivered from each local solar agent was tuned properly within the three operational periods. By comparison with Figs. 16 and 17 of Case 1, it can be seen that air temperature does not affect the total output power of the PV farm as much as solar irradiance does. Additionally, the output factor $g_{1}(k)$ of the proposed FLC can be changed online, as described in Fig. 23.

As shown in Fig. 24, battery banks installed in the three PV agents are governed to supply power in three periods (roughly, [0 s, $1.8 \mathrm{~s}$ ], [30.7 s, $34.4 \mathrm{~s}$ ], and [39.9 s, $45 \mathrm{~s}$ ]) and to absorb power in three periods (roughly, [1.8 s, $7.55 \mathrm{~s}$ ], [34.4 s, $38.75 \mathrm{~s}]$, and [39.1 s, $39.9 \mathrm{~s}]$ ). By using the suggested coordinated strategy, the battery banks are operated in a way that significantly reduces errors in grid frequency, particularly when the air temperature and AGC parameters change unexpectedly at $t=20$ (s) and $t=30(\mathrm{~s})$. 


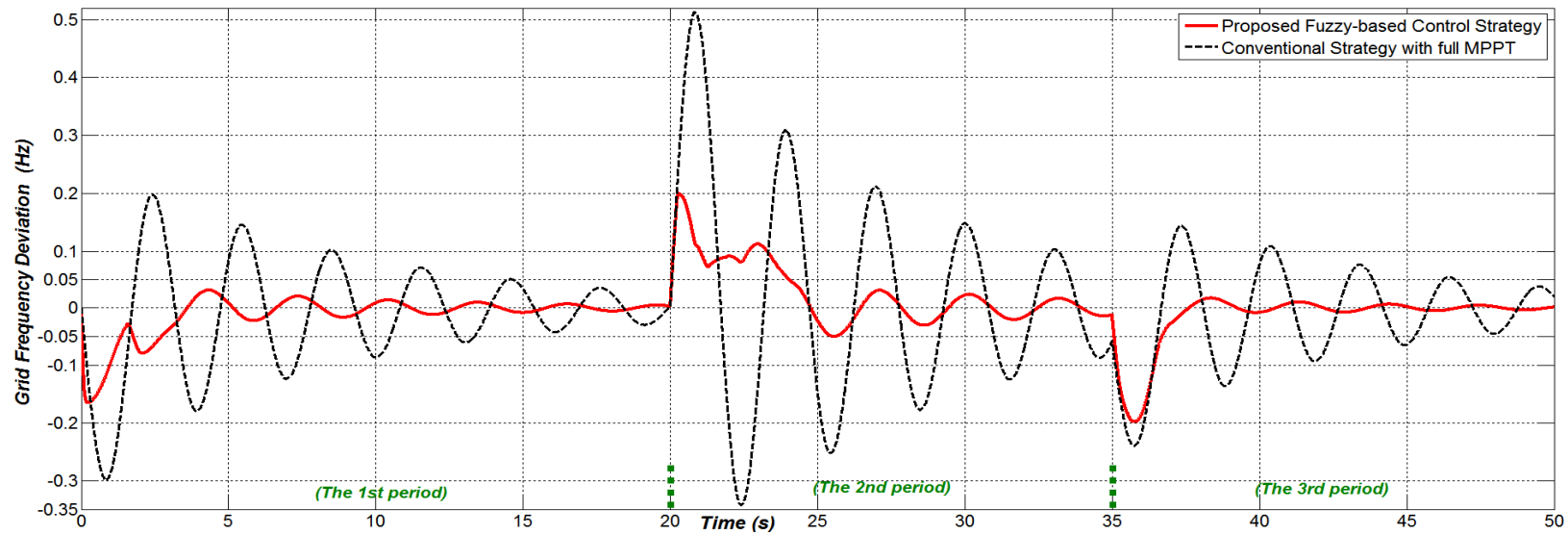

Fig. 15. Grid frequency deviation $E f_{G}(k)$ with three different operational periods in Case 1.

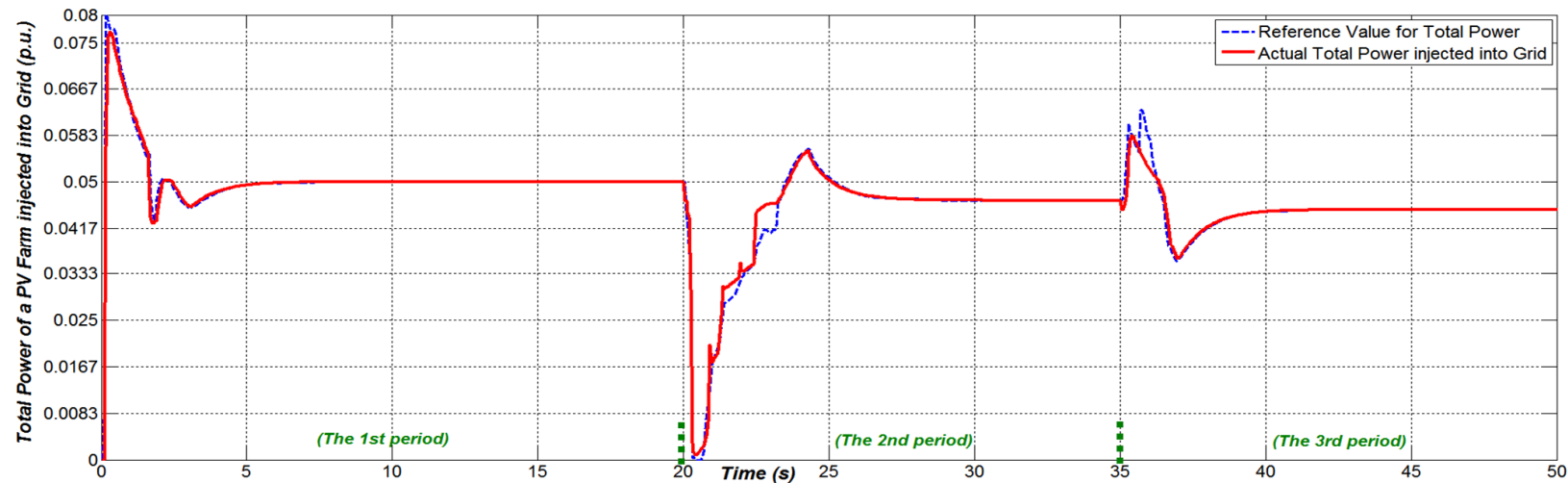

Fig. 16. Total active power injected from the PV farm into grid under the proposed strategy, Case 1.

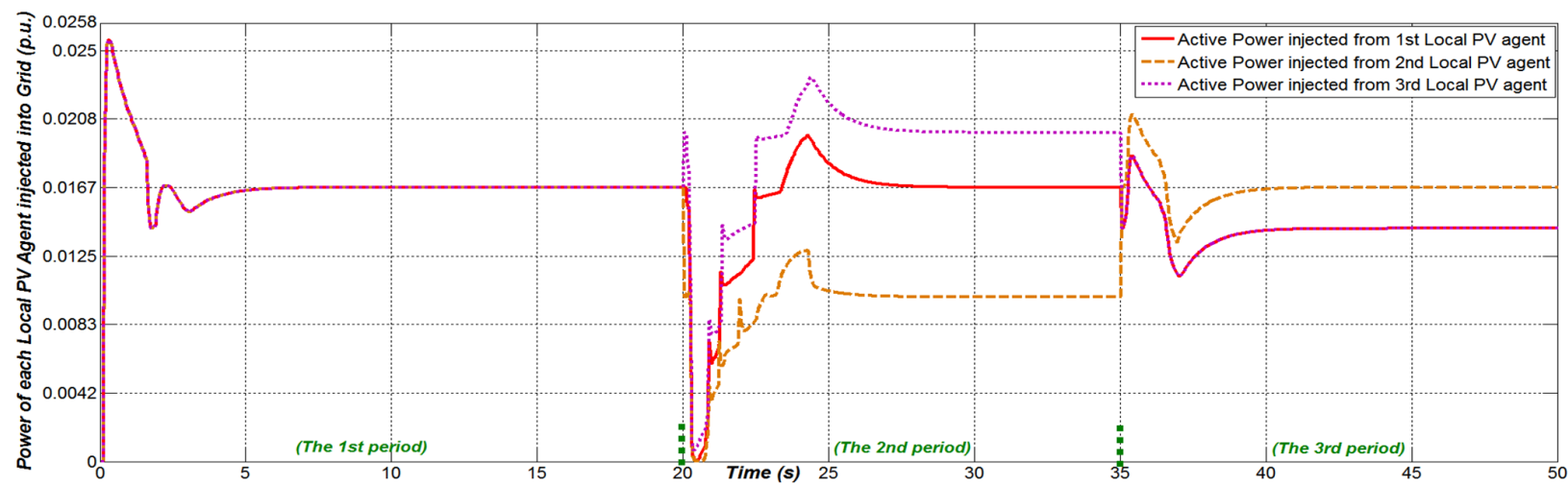

Fig. 17. Individual active power injected from each local agent in the PV farm under the proposed strategy, Case 1.

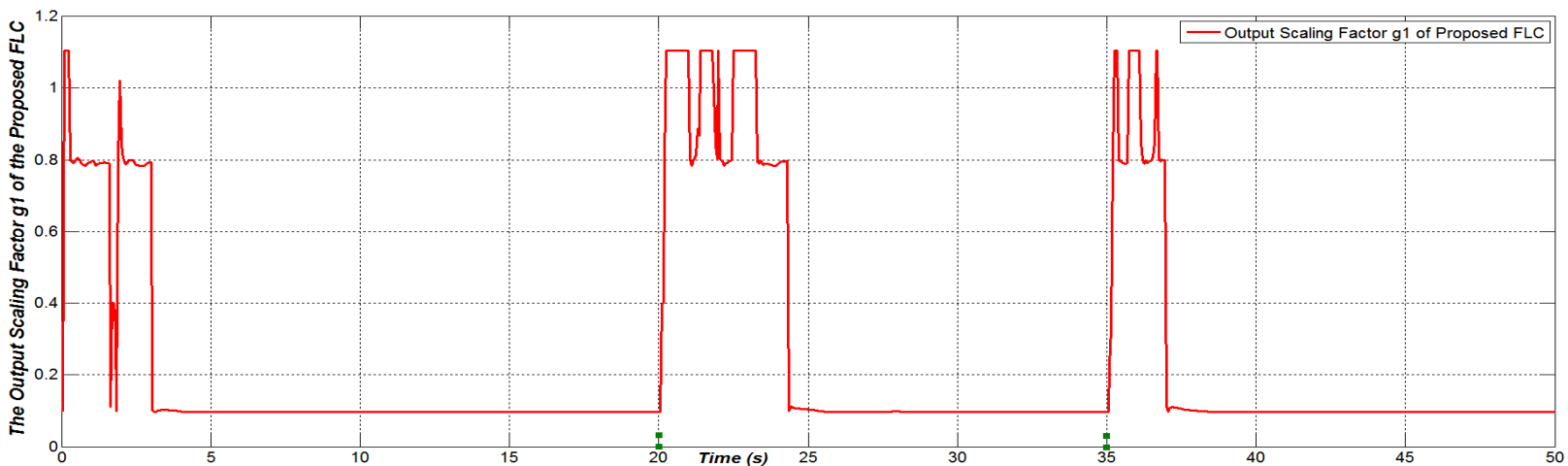

Fig. 18. Changeable output factor $g_{1}(k)$ of the proposed FLC scheme, Case 1 . 


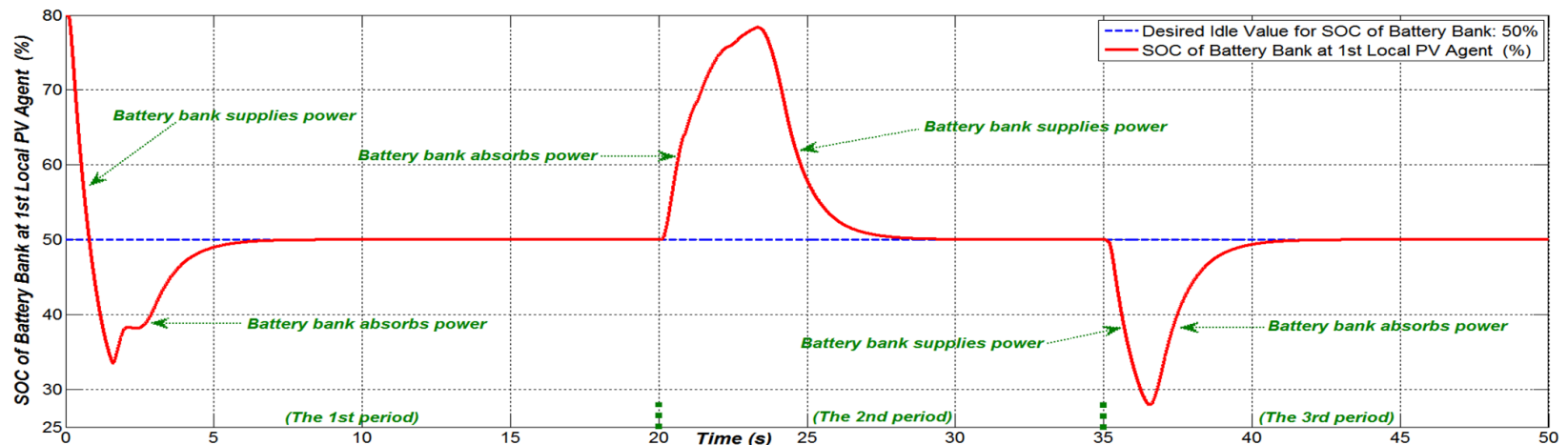

(a) SOC of battery bank at the first local agent

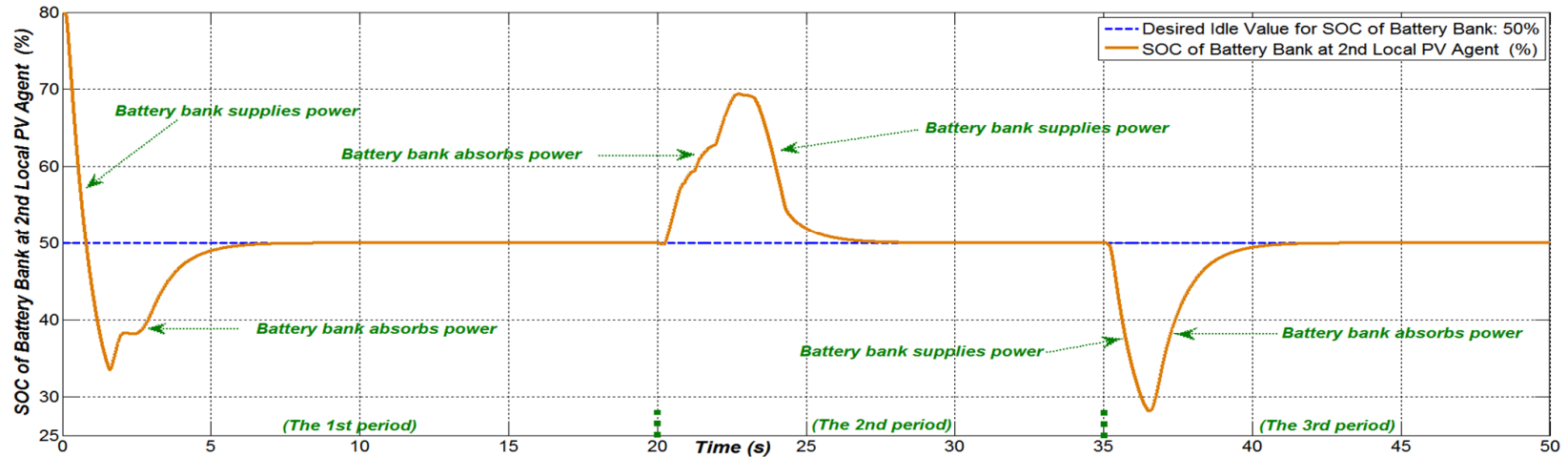

(b) SOC of battery bank at the second local agent

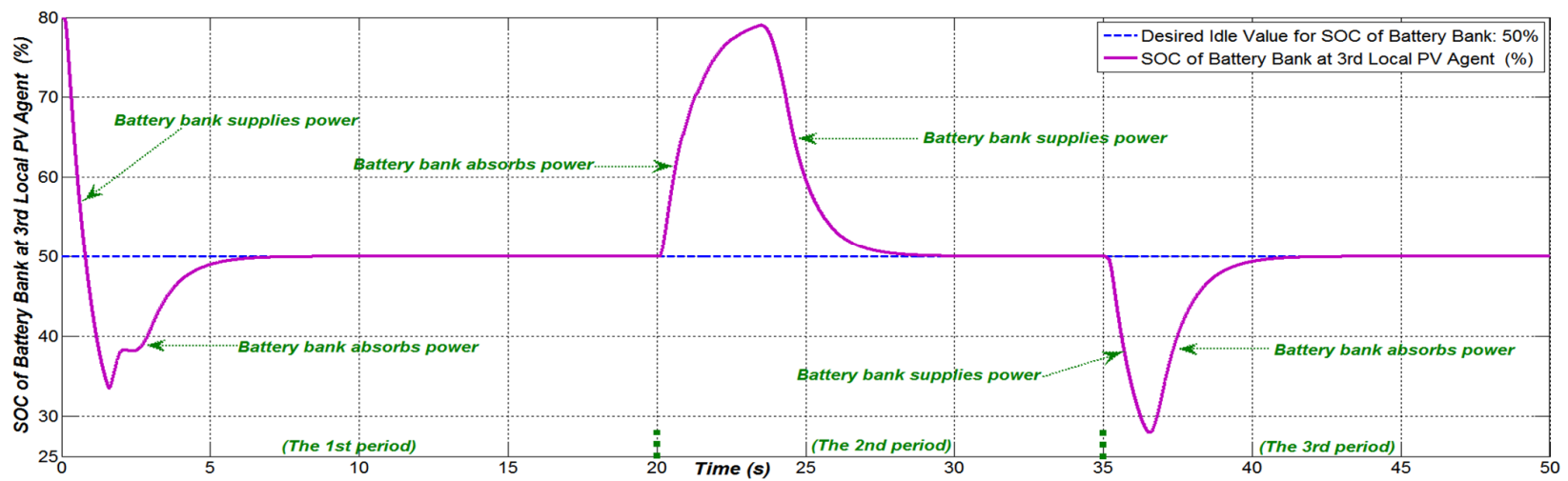

(c) SOC of battery bank at the third local agent

Fig. 19. SOC of battery banks at three local agents in the PV farm under the proposed strategy, Case 1.

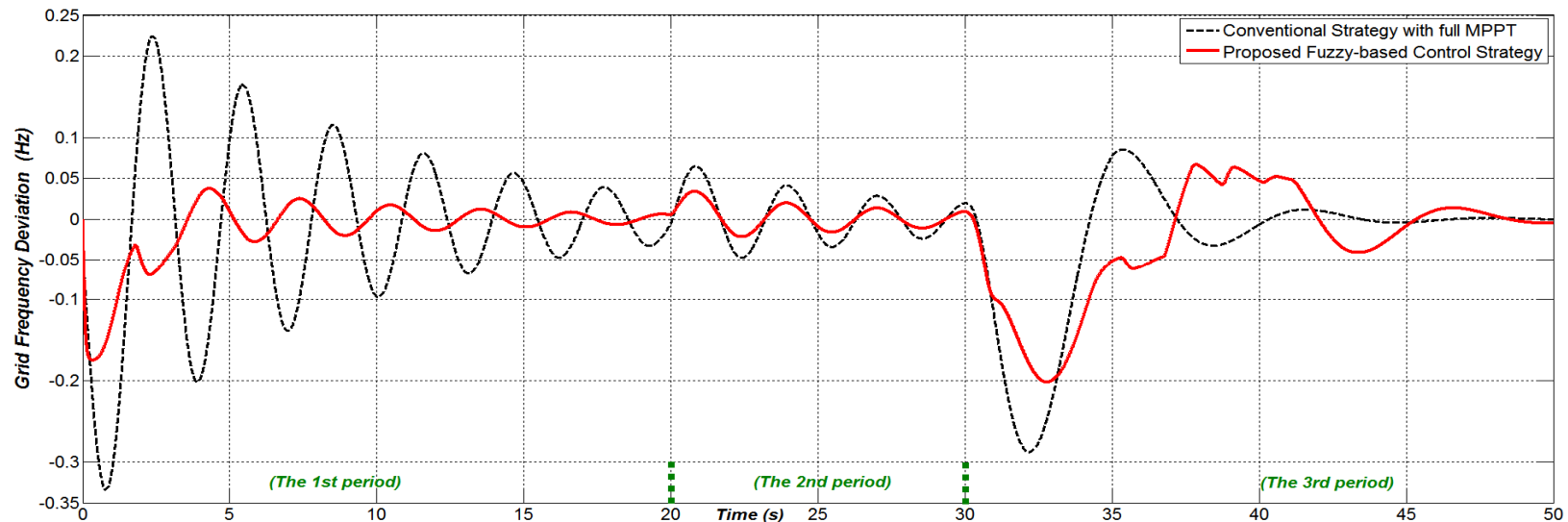

Fig. 20. Grid frequency deviation $E f_{G}(k)$ during three different operational periods, Case 2. 


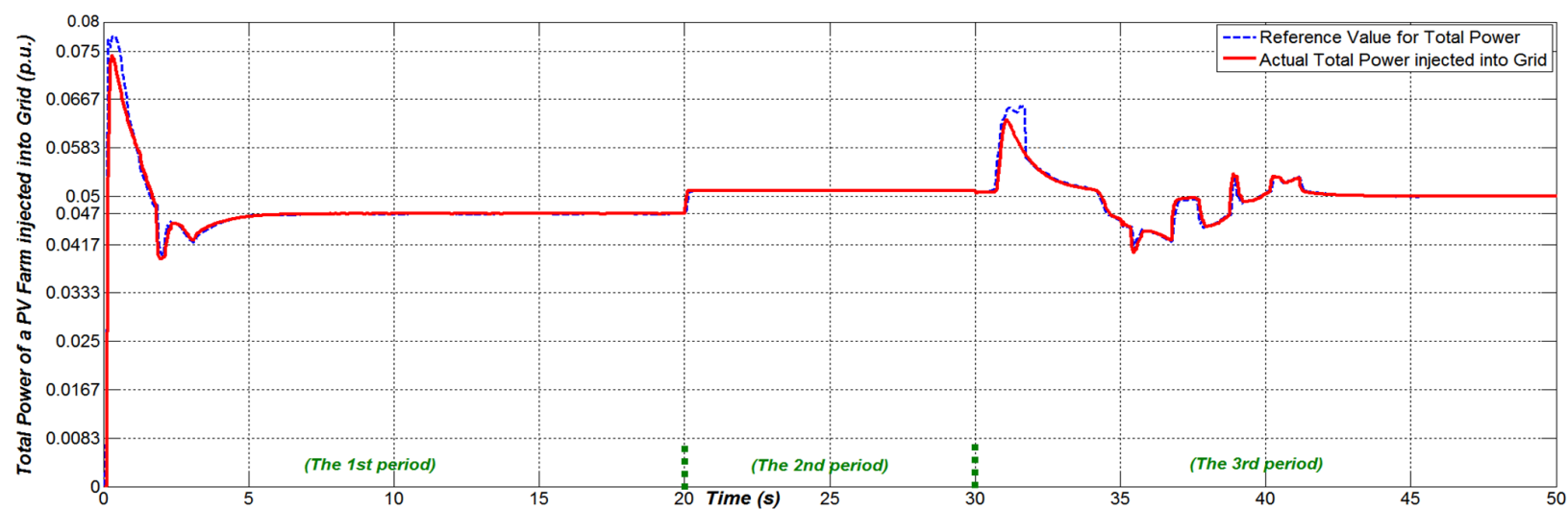

Fig. 21. Total active power injected from the PV farm into grid under the proposed strategy, Case 2.

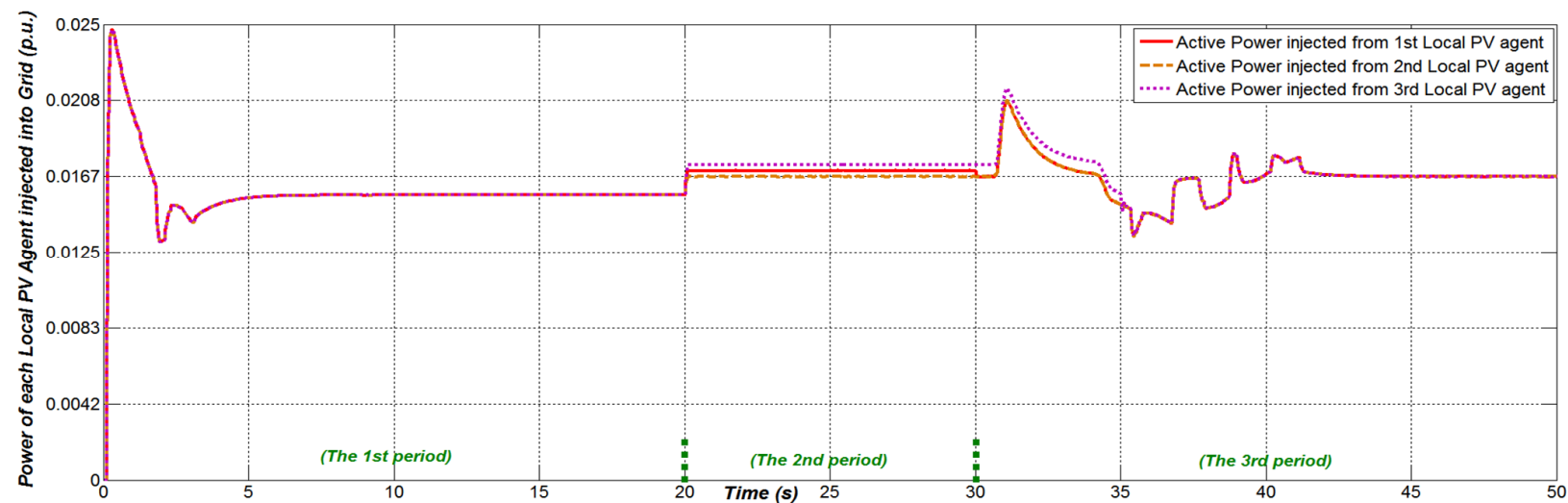

Fig. 22. Individual active power injected from each local agent in the PV farm under the proposed strategy, Case 2.

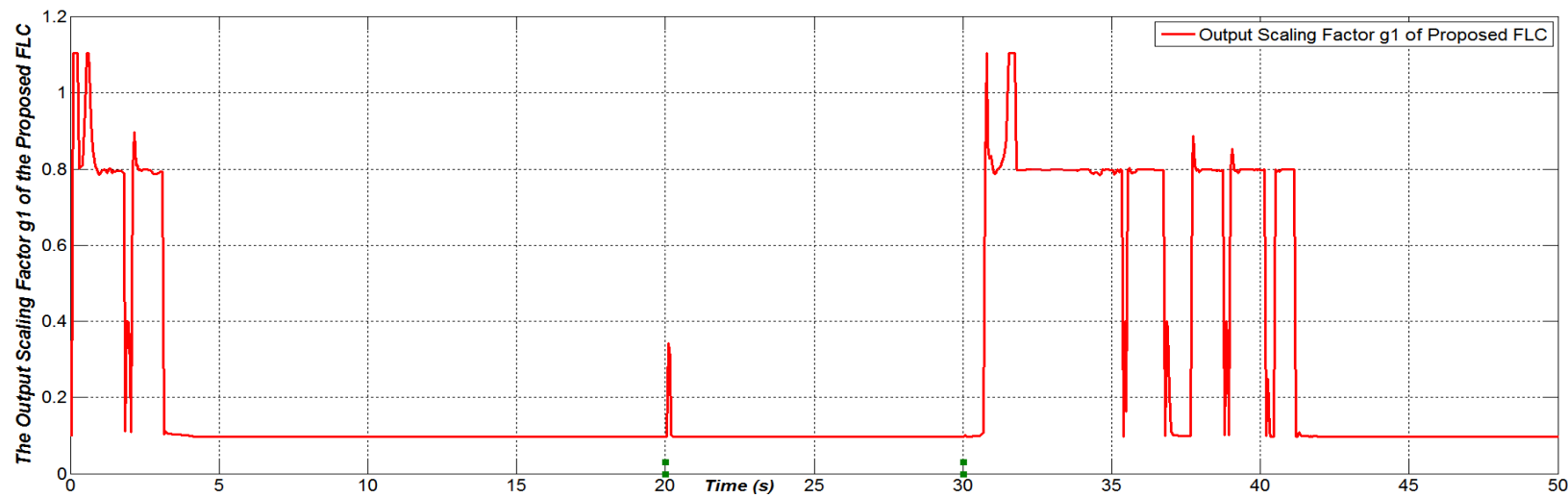

Fig. 23. The changeable output factor $g_{1}(k)$ of the proposed FLC scheme, Case 2.

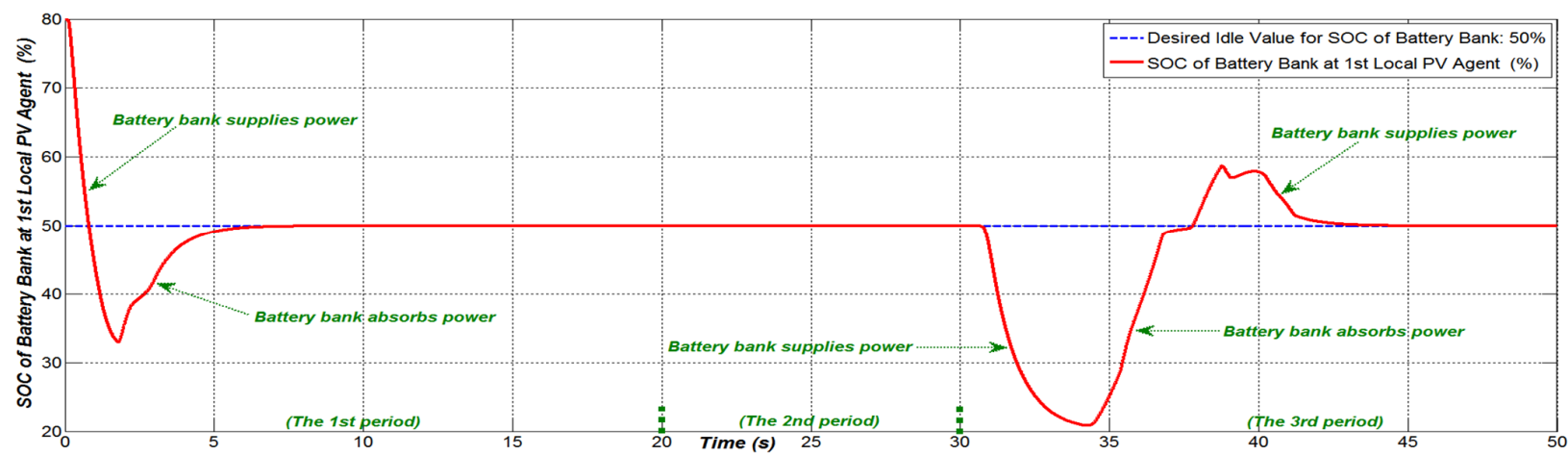

(a) SOC of battery bank at the first local agent 


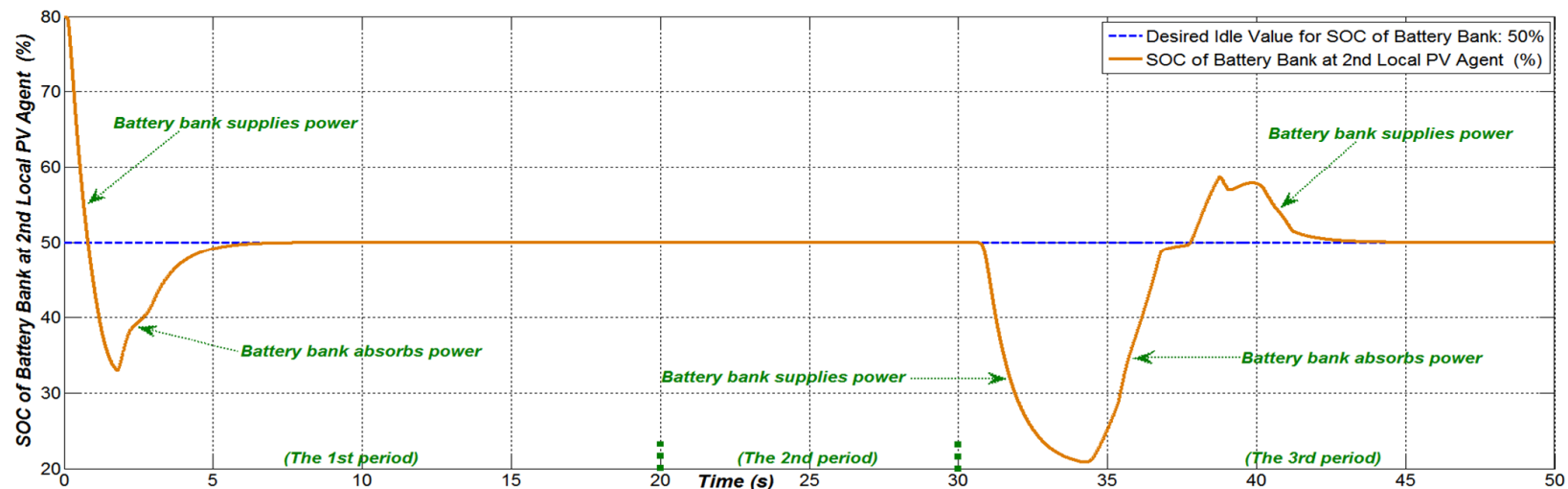

(b) SOC of battery bank at the second local agent

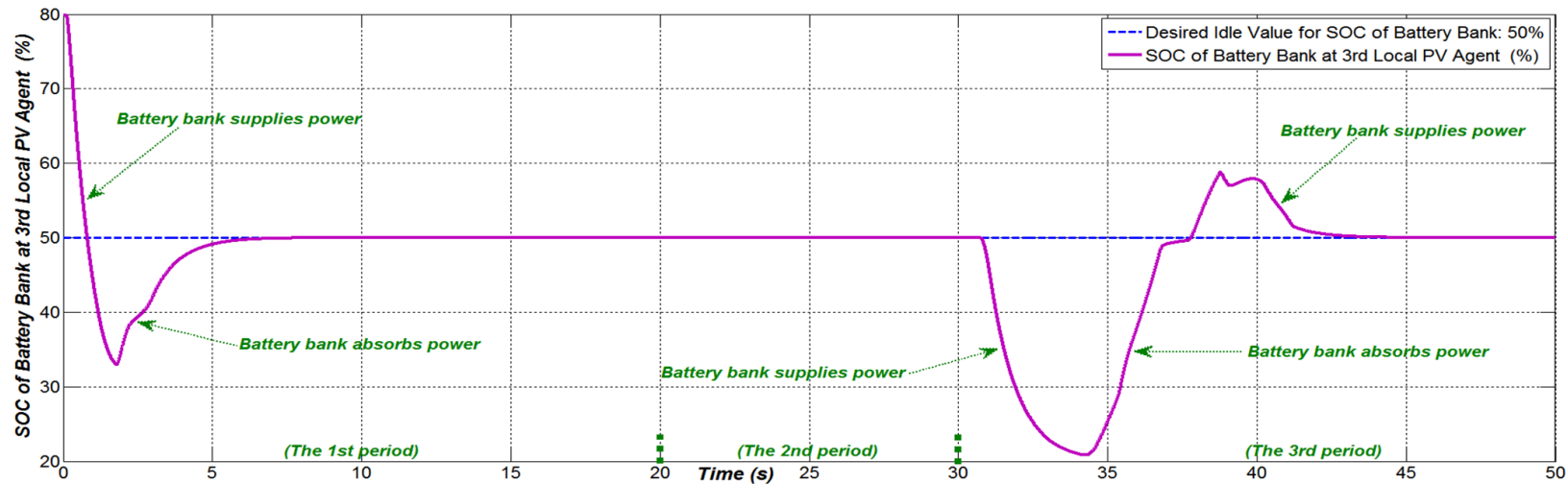

(c) SOC of battery bank at the third local agent

Fig. 24. SOC of battery banks at three local agents in the PV farm under the proposed strategy, Case 2.

\subsection{Case 3: Four-area power system with four PV farms connected}

Table 7. Parameters of the four-area power system in Fig. 25.

\begin{tabular}{c} 
Parameter values of the power Area 1 \\
\hline$B_{1}=14 ; K i_{1}=0.4 ; R_{1}=0.1 ; T_{G 1}=0.2 ; T_{T 1}=0.5 ;$ \\
$M_{1}=3.5 ; D_{1}=1$
\end{tabular}

Table 8. Changes of AC system load by area for Case 3.

\begin{tabular}{|c|c|c|c|}
\hline \multirow{2}{*}{$\begin{array}{c}\text { Four areas } \\
\text { with four } \\
\text { PV farms }\end{array}$} & \multicolumn{3}{|c|}{ Three operational time periods } \\
\cline { 2 - 4 } & $\begin{array}{l}\text { 1st period } \\
t=[0,20) \mathrm{s}\end{array}$ & $\begin{array}{c}\text { 2nd period } \\
t=[20,45) \mathrm{s}\end{array}$ & $\begin{array}{c}\text { 3rd period } \\
t=[45,70] \mathrm{s}\end{array}$ \\
\hline Area 1 (farm 1) & 0.082 p.u. & 0.0263 p.u. & 0.0632 p.u. \\
\hline Area 2 (farm 2) & 0.082 p.u. & 0.0402 p.u. & 0.082 p.u. \\
\hline Area 3 (farm 3) & 0.082 p.u. & 0.082 p.u. & 0.0656 p.u. \\
\hline Area 4 (farm 4) & 0.082 p.u. & 0.082 p.u. & 0.059 p.u. \\
\hline Solar radiations & $1 \mathrm{~kW} / \mathrm{m}^{2}$ & $0.933 \mathrm{~kW} / \mathrm{m}^{2}$ & $1.067 \mathrm{~kW} / \mathrm{m}^{2}$ \\
\hline & (same radiation values for four areas) \\
\hline Temperatures & \multicolumn{3}{|c|}{ fixed at $25^{\circ} \mathrm{C}$} \\
\hline
\end{tabular}

In this third case, the proposed coordinated control strategy is implemented for four large-scale PV farms, which are connected in the four-area power system shown schematically in Fig. 25.

Weather conditions in all four areas are similar, and the grid power capacity at each area is 6 (MW). However, the operating parameters of grids at the areas are fairly different (see Table 7) and AC system loads change (see Table 8). It is noted that 1 (p.u.) still corresponds to a normalized power of 6 (MW). The main goal of this test case is to examine performance of the strategy and interaction effects between the power areas.

The performances of the four-area power system under the proposed strategy are presented in Figs. 26 to 32. Wherein, Figs. 26 to 29 show the grid frequency deviations 


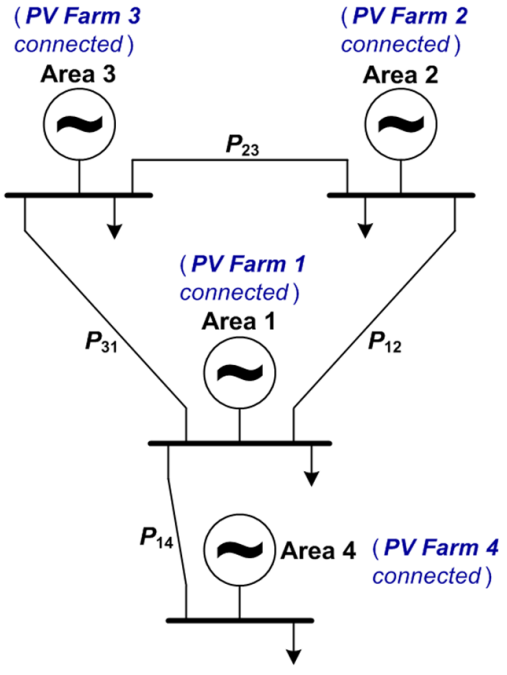

(a) Study case of a four-area power system with four PV farms connected

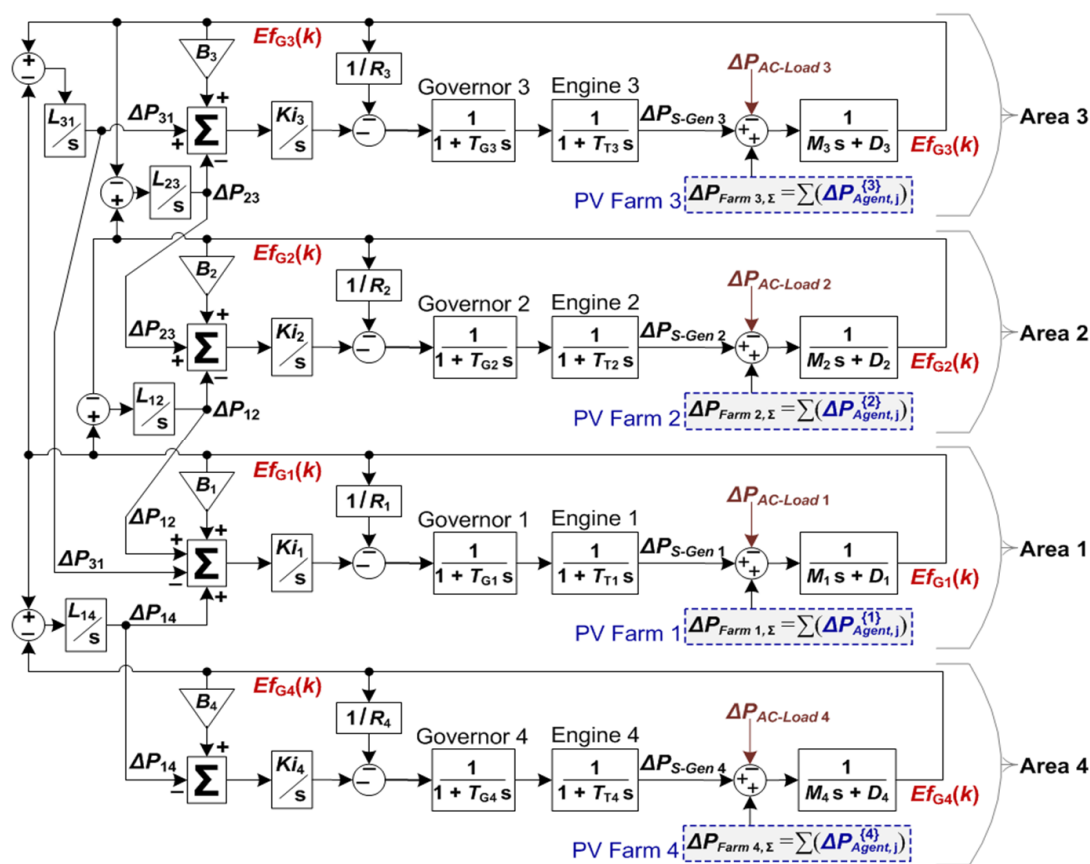

(b) Detailed diagram in simulation of the four-area power system in (a)

Fig. 25. Four-area system with four PV farms connected and tie-line power flows, Case 3.

under the proposed strategy for all four areas. All areas are stabilized to the tolerable standard range of $\pm 0.2(\mathrm{~Hz})$ and rapidly reach to the steady-state range of $\pm 0.05(\mathrm{~Hz})$. In contrast, under the conventional strategy, tie-line power flows between the four areas heavily affect the grid frequency, causing large and fairly complicated errors.

For example, at the beginning of the third operation period, $t=45(\mathrm{~s})$, the AC system load in Area 4 is significantly reduced from 0.082 (p.u.) to 0.059 (p.u.), and the solar radiation rises from $0.9333\left(\mathrm{~kW} / \mathrm{m}^{2}\right)$ to 1.067 $\left(\mathrm{kW} / \mathrm{m}^{2}\right)$ as given in Table 8 . As a result, the grid frequency deviation at this area is predicted to increase noticeably. With the conventional strategy, because the tie-line power $P_{14}$ (between Area 1 and Area 4) has a negative value, as described in Fig. 32(a), this tie-line power is presently transmitted from Area 4 to Area 1 to reduce the grid frequency deviation at Area 4. However, the transmission speed of $P_{14}$ is not fast enough to resolve this issue. Hence, the frequency deviation reaches the peak point $b_{1}$ of approximately $0.45(\mathrm{~Hz})$, which far beyond the upper limiting value of $0.2(\mathrm{~Hz})$ as shown in Fig. 29. Meanwhile, with the proposed strategy, the emergency condition expressed in (16) and (17) is now activated to immediately reduce the total power of the grid at Area 4 as presented in Figs. 30(d) and 31(d). In addition, part of this area's power is transmitted to Area 1 because $P_{14}$ has a negative value as depicted in Fig. 32(b). All this facilitates rapidly reducing the total power at Area 4, the ultimate goal of which is to bring the frequency deviation to the peak point $c_{1}$, which is still below the upper limiting value of $0.2(\mathrm{~Hz})$.

In the next cycle, because of effects of AGC used in Area 4 and the tie-line power $P_{14}$, the frequency deviation at Area 4 with the conventional strategy reaches the point $b_{2}$, which has a value of about $-0.17(\mathrm{~Hz})$ as given in
Fig. 29. Meanwhile, with the proposed strategy, the battery banks of the PV farm in Area 4 are still absorbing power (see Fig. 31(d)) and the tie-line power $P_{14}$ is still being transmitted at a high rate from Area 4 to Area 1. Hence, the grid frequency deviation is decreased from the peak point $c_{1}$ to the new peak point $c_{2}$, which has a value of about $0.11(\mathrm{~Hz})$. From the above observations, it can be seen that the grid frequency deviation at Area 4 with the suggested strategy is always inside the acceptable range of $\pm 0.2(\mathrm{~Hz})$ and is smaller in magnitude than would be the case with the conventional simple strategy.

For the proposed strategy, Fig. 30 shows the individual total power supplied from each PV farm to the grid. The actual output power values were controlled to closely track the reference values. The four PV farms have the same design structure and weather conditions (as given in Tables 7 and 8), but the areas have differences in parameters of the grids, system loads, and locations in the power network (see Fig. 25), so the total power injected into the grid from each farm is suitably adjusted with a different amount.

The SOC values for the battery banks at the first agent of each PV farm are illustrated in Fig. 31. All the SOC values are governed to within the safe ratio value range 20 $80 \%$ during the operation time, and regulated closely to the desired idle ratio value of $50 \%$ at the steady state. In fact, the battery banks are driven to supply or absorb power as needed to help flexibly adjust the total power injected into the grid from PV farms. Finally, with the proposed strategy, the tie-line power flows between the four areas were relatively well stabilized as shown in Fig. 32(b). These responses are better than the ones with the conventional strategy described in Fig. 32(a). 


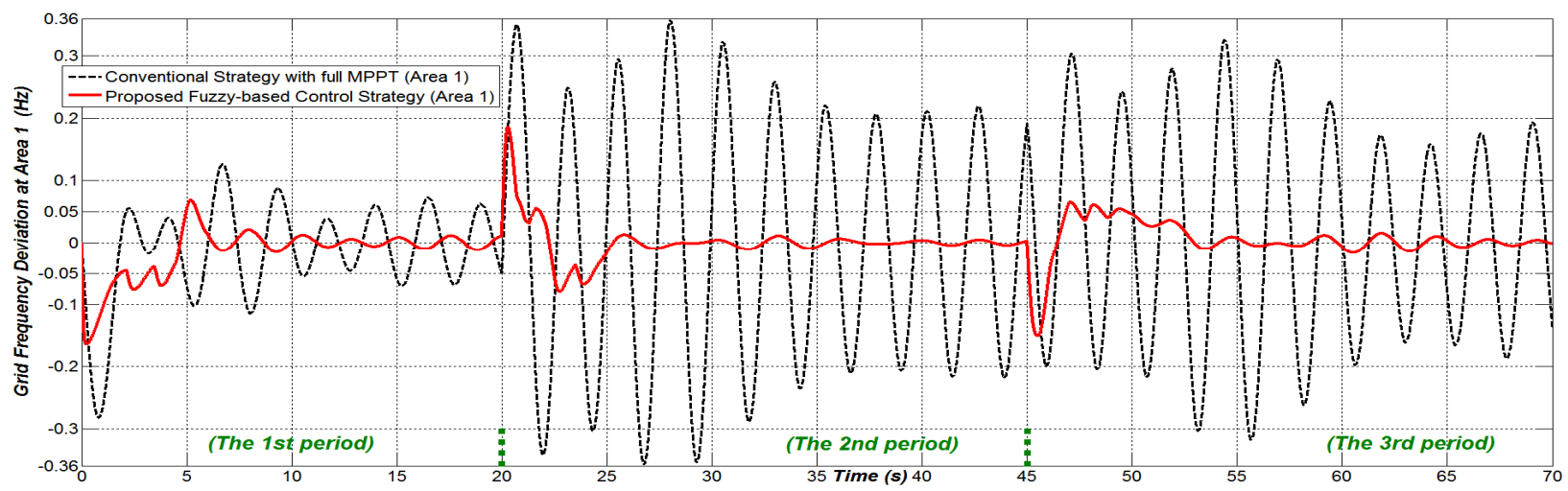

Fig. 26. Grid frequency deviation at Area $1 E f_{G 1}(k)$ for three different operational periods, Case 3.

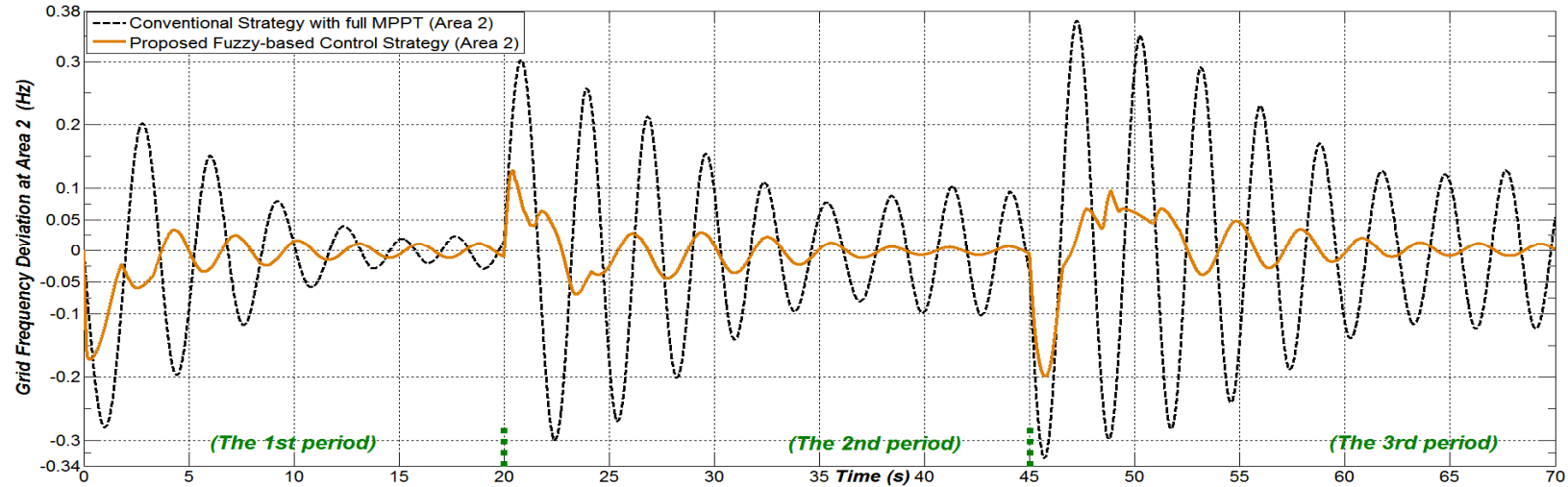

Fig. 27. Grid frequency deviation at Area $2 E f_{G 2}(k)$ for three different operational periods, Case 3.

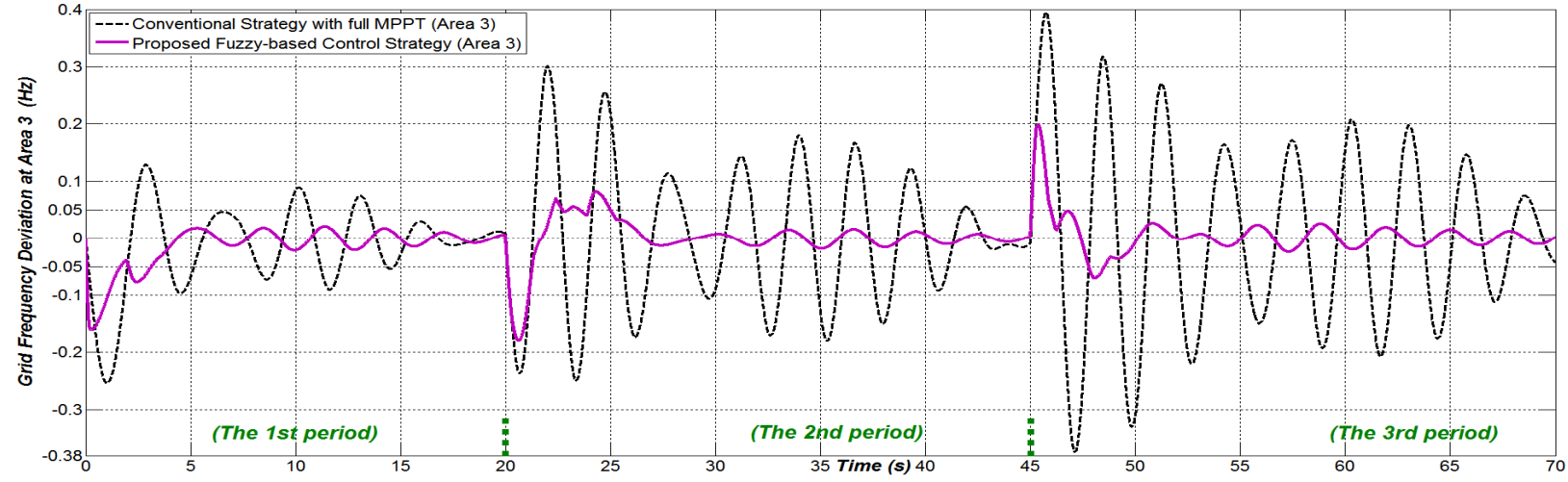

Fig. 28. Grid frequency deviation at Area $3 E f_{G 3}(k)$ for three different operational periods, Case 3.

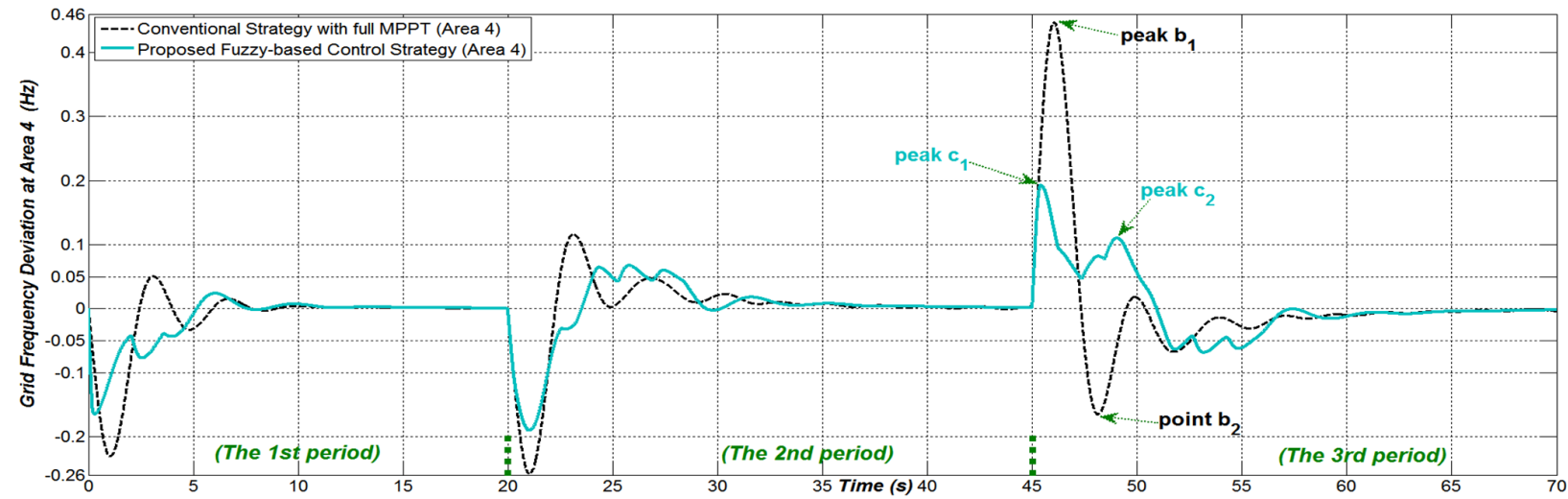

Fig. 29. Grid frequency deviation at Area $4 E f_{G 4}(k)$ for three different operational periods, Case 3. 


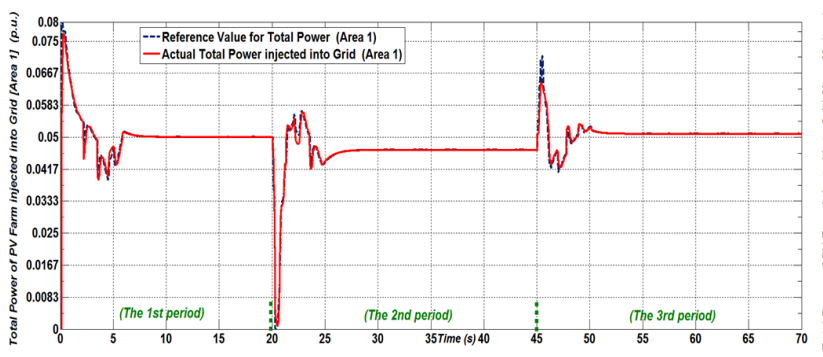

(a) Total power from the PV farm of Area 1

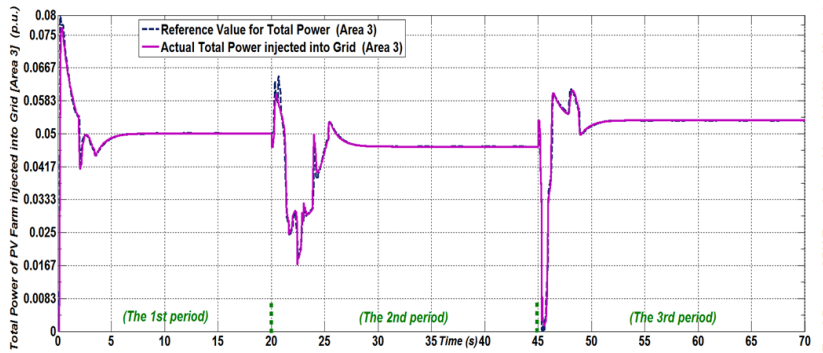

(c) Total power from the PV farm of Area 3

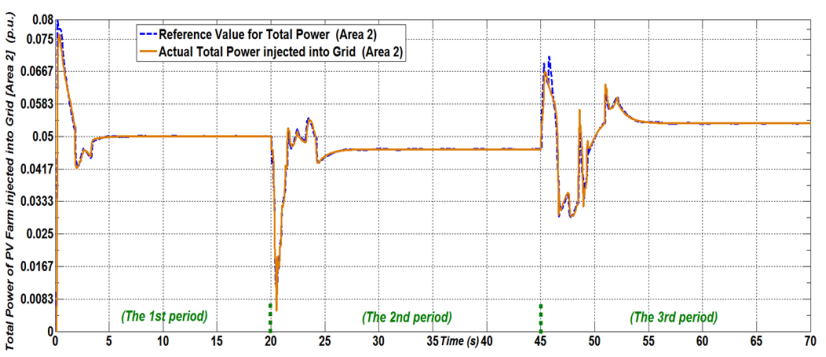

(b) Total power from the PV farm of Area 2

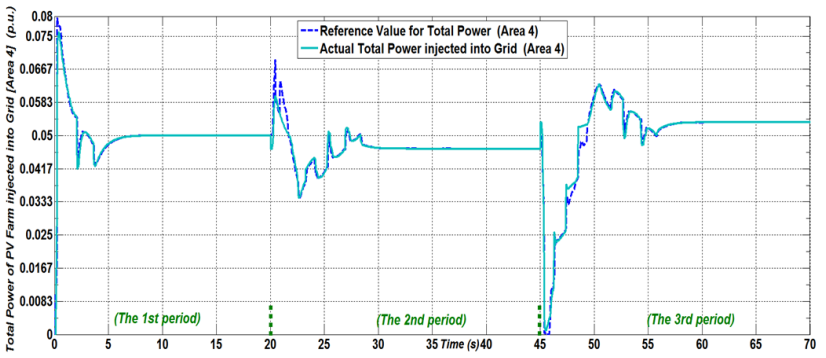

(d) Total power from the PV farm of Area 4

Fig. 30. Total active power injected from the PV farm of each area under the proposed strategy, Case 3.

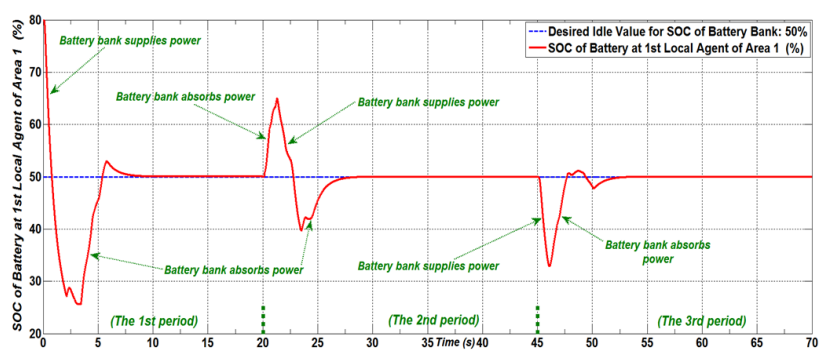

(a) SOC of battery bank at first agent of PV farm in Area 1

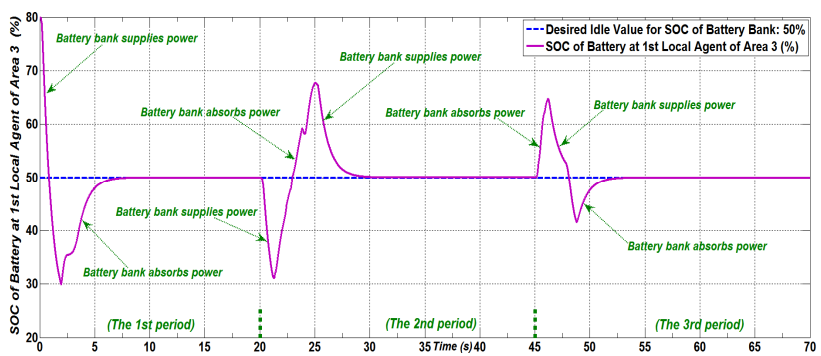

(c) SOC of battery bank at first agent of PV farm in Area 3

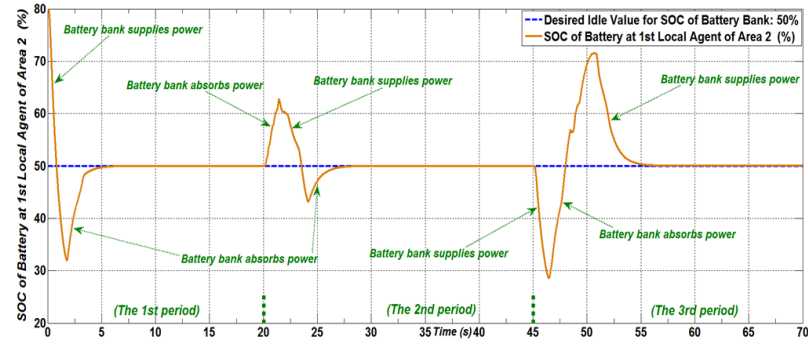

(b) SOC of battery bank at first agent of PV farm in Area 2

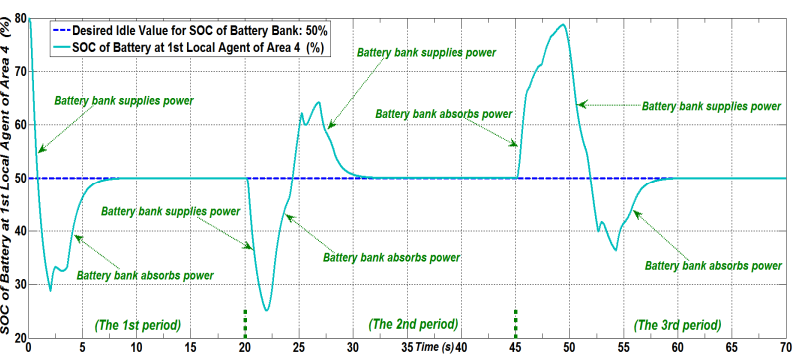

(d) SOC of battery bank at first agent of PV farm in Area 4

Fig. 31. SOC of battery banks at local agents for four PV farms under the proposed strategy, Case 3.

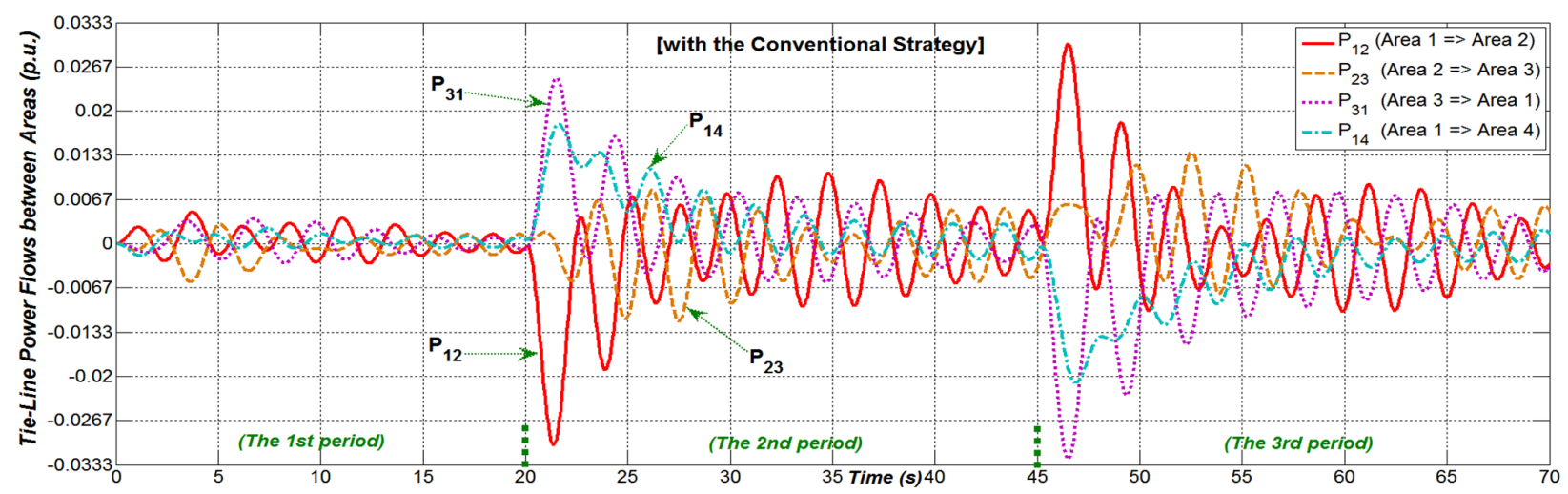

Fig. 32(a). Tie-line active power flows between four areas with the conventional strategy in Case 3. 


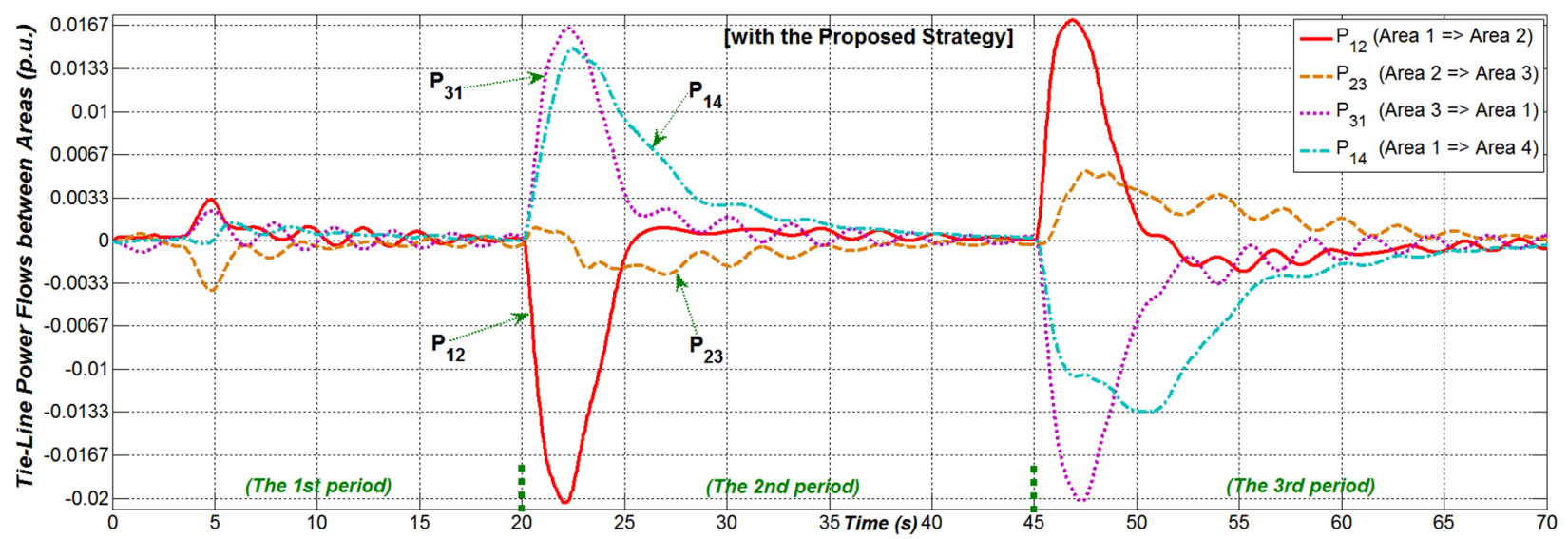

Fig. 32(b). Tie-line active power flows between four areas with the proposed strategy in Case 3.

\section{Discussion}

This section presents several additional issues that should be noted when implementing the proposed coordinated control strategy in large-scale PV farms.

The effectiveness and stability of the suggested control strategy are highly dependent on the individual reference value $\beta_{\text {Agent, } j}^{*}(k)$, which is sent from the central controller to the local controller of each PV agent in a solar farm. If $\beta_{\text {Agent }, j}^{*}(k)$ is much larger than the real maximum power capacity of the $j$ th local PV agent, then the actual total power injected into the grid from the solar farm (including all local agents) $\beta_{F a r m, \Sigma}^{\text {Meas }}(k)$ will surely be smaller than its computed reference value $\beta_{\text {Farm }, \Sigma}^{*}(k)$. This will noticeably degrade the performance in stabilizing the grid frequency. In Section 3 of this paper, some limits were set in (15) for $\beta_{\text {Farm }, \Sigma}^{*}(k)$ and in (22) for $\beta_{\text {Agent }, j}^{*}(k)$. These limits are necessary and helpful in eliminating this problem. To ensure (15) and (22) result in the correct settings, the predicted maximum power from PV arrays $\beta_{P V o n l y, j}^{\text {Pre }}(k)$ and the percentage of total power loss $\mu_{L, j}$ in each the local agent should be determined relatively correctly.

As described in Fig. 1(a), at the local agent, the individual MPPT controllers are separately implemented for each DC-DC converter connected with the PV array. Because of this, the negative impact of simple partial shading conditions on the output total power of the PV farm is reduced remarkably. Despite this, large-scale solar farms involve many PV arrays installed in a big area, and so partial shading conditions may be quite complicated for some PV arrays. Thus, short-term prediction for climate (see [35]) or an enhanced MPPT algorithm (see [36],[37]) could be employed to integrate specialized functions to account for the complex partial shading conditions better than the conventional MPPT algorithm shown in Figs. 6 and 7 does. The purpose is to better predict the maximum power possible from all PV arrays $\beta_{P V o n l y, j}^{\text {Pre }}(k)$ for each local agent even under partial shading situations, and the ultimate goal is to boost accuracy in predicting the total capacity $\beta_{\text {Farm }, \Sigma}^{\text {Pre,Max,TrS }}(k)$ for the PV farm.
As shown in (20), (21), and (26), the value of $\mu_{L, j}$ is the percentage of estimated total power loss (including all losses due to power-electronic converters, the efficiency of MPPT algorithm implemented in DC for each local agent DC converters, errors in the prediction module, and a minor amount of power for backup) relative to the nominal total capacity for each local PV agent. In fact, it is difficult to clearly determine the most appropriate value for $\mu_{L, j}$. Normally, the value of $\mu_{L, j}$ can be estimated to be within the range $0-15 \%$ for typical grid-connected PV farms. In the simulations considered in this study, $\mu_{L, j}$ was set to $5 \%$ as a small value to highlight the operation of the proposed fuzzy-based strategy. However, for real PV farms, $\mu_{L, j}$ could be larger than $5 \%$ depending on system configuration and operating modes.

In this study, local battery banks are chosen for use in the PV farm because of the following observations and reviews. First, the maintenance, repair, and/or replacement for a kW-class battery bank at each local solar agent in cases of sudden malfunction are often more convenient and flexible than with a grid-scale MW-class battery storage. Second, if several local solar agents are added into the physical PV farm (or several agents are removed), the number of agents $N$ will change, as will the total capacity of the PV farm. However, the proposed coordinated strategy in this paper is still suitably adaptive because of its unique design structure as shown in Figs. 4(a) and 8 and in (11). No crucial modifications are required for the proposed strategy in these cases. This means that using local battery banks in solar agents would help attain a better performance in the coordinated control and management for a large-scale PV farm. Third, studies on large-scale PV power systems that use distributed battery banks were presented in [1],[4],[38], [39]. There, the use of local battery banks was shown to be efficient in improving the power quality. Moreover, in recent media reports [40]-[42], distributed battery storage has been predicted to increase total power capacity significantly and to play a major role in the energy market of the near future. Also, real large-scale megawatt PV systems combined with residential and commercial areas, in which each solar power unit of the PV systems directly supplies electricity for one or more residential houses or for a commercial building, have 
been developed and implemented [43]-[46]. Last, the design structure for a PV farm with distributed battery banks at agents is the starting point for future work in an on-going research project by the authors, which is presented briefly at the end of the next section.

\section{Conclusion}

This paper has introduced a hierarchical coordinated control strategy based on fuzzy logic to support efficient regulation of grid frequency for large-scale solar farms. These solar farms are expected to utilize battery banks to manage appropriately the energy obtained from all PV arrays installed in the farms. In the illustrated solar farm, the control strategy uses a central controller with the newly proposed automatic-tuning FLC scheme, comprising a changeable output scaling factor $g_{1}(k)$, to set accurately the reference value of total power needed to inject into the grid from the farm. The central controller then coordinates the individual reference values for the local controller installed at each PV power agent. At each PV agent, the power-electronic converters are driven by the local controller as needed to deliver the proper amount of active power to the grid for maintaining the frequency deviation to within an acceptable range, here $\pm 0.2(\mathrm{~Hz})$ in the transient state and $\pm 0.05(\mathrm{~Hz})$ at the steady state. Furthermore, a special control mode is included in the suggested strategy to stabilize rapidly the grid frequency under emergency circumstances, bringing it into the acceptable range above.

Three test cases have confirmed the advantages of the proposed fuzzy-based strategy. These advantages are in supplying the correct amount of active power from the solar farms to the grid and in regulating the grid frequency, and stabilizing the SOC of the battery banks at local PV agents. The SOC is kept in the safe range $20-80 \%$ during operation and near $50 \%$ at the steady state.

The simulation results for the presented strategy have demonstrated superior performance relative to the conventional strategy, which is based on full MPPT mode. This remains the case even when solar irradiance, air temperature, AC system load, and two parameters of the AGC change abruptly and markedly. A four-area network, in which four large-scale PV farms are installed and the tie-line power flows between the areas are controlled, has been considered as a test case. The results from this case have shown the good adaptability of the proposed strategy.

In future work, a decentralized mechanism for grid-connected large-scale PV farms combined with many residential houses and commercial buildings will be researched and designed. In a PV farm, each local solar agent will have a nominal PV power of around $100(\mathrm{~kW})$ plus an $80-\mathrm{kW}$ battery bank to provide electricity to a group of several houses or a commercial building. With this mechanism, key issues on the grid-frequency regulation, optimization of economic benefits for users in residential houses and commercial buildings (which are supplied power directly from the local solar-plus-battery agents of the PV farms), and maximization of social welfare will be considered and resolved.

\section{Acknowledgment}

The authors would like to express the sincere thanks to the editors and reviewers of the journal for your very helpful comments on this paper.

This work was supported by JST-CREST.

\section{References}

[1] M. Datta, H. Ishikawa, H. Naitoh, and T. Senjyu, "LFC by Coordinated Virtual Inertia Mimicking and PEVs in Power Utility with MW-class Distributed PV Generation," Proceedings of 2012 IEEE Workshop on Control and Modeling for Power Electronics, Japan (2012).

[2] M. Datta, T. Senjyu, A. Yona, T. Funabashi, and C. H. Kim, "A Coordinated Control Method for Leveling PV Output Power Fluctuations of PV-Diesel Hybrid Systems Connected to Isolated Power Utility," IEEE Transactions on Energy Conversion, Vol. 24, No. 1, pp. 153-162 (2009).

[3] M. Datta, T. Senjyu, A. Yona, T. Funabashi, and C. H. Kim, "A Frequency-Control Approach by Photovoltaic Generator in a PV-Diesel Hybrid Power System," IEEE Transactions on Energy Conversion, Vol. 26, No. 2, pp. 559-571 (2011).

[4] M. Datta, T. Senjyu, A. Yona, T. Funabashi, and C. H. Kim, "Fuzzy Control of Distributed PV Inverters/Energy Storage Systems/Electric Vehicles for Frequency Regulation in a Large Power System," IEEE Transactions on Smart Grid, Vol. 4, No. 1, pp. 479-488 (2013).

[5] Y. Ghiassi-Farrokhfal, F. Kazhamiaka, C. Rosenberg, and S. Keshav, "Optimal Design of Solar PV Farms with Storage," IEEE Transactions on Sustainable Energy, Vol. 6, No. 4, pp. 1586-1593 (2015).

[6] K. Sun, L. Zhang, Y. Xing, and J. M. Guerrero, "A Distributed Control Strategy Based on DC Bus Signaling for Modular Photovoltaic Generation Systems with Battery Energy Storage," IEEE Transactions on Power Electronics, Vol. 26, No. 10, pp. 3032-3045 (2011).

[7] N. Kakimoto, S. Takayama, H. Satoh, and K. Nakamura, "Power Modulation of Photovoltaic Generator for Frequency Control of Power System," IEEE Transactions on Energy Conversion, Vol. 24, No. 4, pp. 943-949 (2009).

[8] H. Xin, Y. Liu, Z. Wang, D. Gan, and T. Yang, "A New Frequency Regulation Strategy for Photovoltaic Systems without Energy Storage," IEEE Transactions on Sustainable Energy, Vol. 4, No. 4, pp. 985-993, (2013).

[9] G. Delille, B. François, and G. Malarange, "Dynamic Frequency Control Support by Energy Storage to Reduce the Impact of Wind and Solar Generation on Isolated Power System's Inertia," IEEE Transactions on Sustainable Energy, Vol. 3, No. 4, pp. 931-939 (2012).

[10] H. Bevrani, F. Habibi, P. Babahajyani, M. Watanabe, and Y. Mitani, "Intelligent Frequency Control in an AC Microgrid: Online PSO-Based Fuzzy Tuning Approach," IEEE Transactions on Smart Grid, Vol. 3, No. 4, pp. 1935-1944 (2012). 
[11] H. Bevrani and S. Shokoohi, "An Intelligent Droop Control for Simultaneous Voltage and Frequency Regulation in Islanded Microgrids," IEEE Transactions on Smart Grid, Vol. 4, No. 3, pp. 1505-1513 (2013).

[12] W. Bai, M. R. Abedi, and K. Y. Lee, "Distributed Generation System Control Strategies with PV and Fuel Cell in Microgrid Operation," Control Engineering Practice, In Press, Corrected Proof, 10 pages, Available online 17 February 2016.

[13] N. G. M. Thao and K. Uchida, "A Control Strategy based on Fuzzy Logic for Three-phase Grid-connected Photovoltaic System with Supporting Grid-Frequency Regulation," Journal of Automation Control Engineering, Vol. 4, No. 2, pp. 96-103 (2016), Early available online on 11 June 2015.

[14] N. G. M. Thao and K. Uchida, "A Novel Fuzzy-based Control Strategy for Grid-connected Large-scale Solar Farm with Supporting the Grid-Frequency Regulation," Proceedings of 2015 Asian Control Conference, pp. 224-231, Kota Kinabalu, Malaysia (2015).

[15] N. G. M. Thao, K. Uchida, "Control The Photovoltaic Grid-Connected System Using Fuzzy Logic and Backstepping Approach," Proceedings of 2013 Asian Control Conference, pp. 1-8, Istanbul, Turkey (2013).

[16] R. M. Strzelecki and G. Benysek, Power Electronics in Smart Electrical Energy Networks, Springer (2008).

[17] S. Biegacki and D. VanGompel, "The Application of DeviceNet in process control," ISA Transactions, Vol. 35, No. 2, pp. 169-176 (1996).

[18] H. Saadat, Power System Analysis - Chapter 12: Power system control, WCB/McGraw-Hill (1999).

[19] D. C. Martins, "Analysis of a Three-Phase Grid-Connected PV Power System Using a Modified Dual-State Inverter," ISRN Renewable Energy, Vol. 2013, Article ID 406312, 18 pages.

[20] M. Meinhardt, G. Cramer, B. Burger, and P. Zacharias, "Multi-String-Converter with Reduced Specific Costs and Enhanced Functionality," Solar Energy, Vol. 69, No. 6, pp. 217-227 (2001).

[21] T. Esram and P. L. Chapman, "Comparison of Photovoltaic Array Maximum Power Point Tracking Techniques," IEEE Transactions on Energy Conversion, Vol. 22, No. 2, pp. 439-449 (2007).

[22] KWT-250M60A 250W K-Solar PV panel, KyungWon Co. Ltd. (2012).

[23] E. Skoplaki, and J. A. Palyvos, "On the Temperature Dependence of Photovoltaic Module Electrical Performance: A Review of Efficiency /Power Correlations," Solar Energy, Vol. 83, pp. 614-624 (2009).

[24] M. C. Garcia, and J. L. Balenzategui, "Estimation of Photovoltaic Module Yearly Temperature and Performance Based on Nominal Operation Cell Temperature Calculations," Renewable Energy, Vol. 29, pp. 19972010 (2004).

[25] P. Jirutitijaroen, "Transformer and Per-unit Analysis," EE2022 Electrical Energy Systems - lecture 11, National University of Singapore (2012).

[26] N. G. M. Thao and K. Uchida, "Active and Reactive Power Control Techniques based on Feedback Linearization and Fuzzy Logic for Three-phase
Grid-connected Photovoltaic Inverters," Asian Journal of Control, Vol. 17, No. 5, pp. 1522-1546(2015).

[27] T. J. Ross, Fuzzy Logic with Engineering Applications, 3rd edition, Wiley (2010).

[28] O. Wolkenhauer, "Fuzzy Inference Engines," Control Systems Centre, University of Manchester Institute of Science and Technology, available online at: www.sbi.uni-rostock.de/uploads/tx_templavoila/SBI_ Materials_Fuzzy-Inference_Engines.pdf

[29] A. Notholt, D. Coll-Mayor, and A. Engler, "Setting Up a Grid Simulator," Proceedings of 2006 International Conference on Renewable Energies and Power Quality, Balearic, Spain (2006).

[30] M. Valipour, "Optimization of Neural Networks for Precipitation Analysis in A Humid Region to Detect Drought and Wet Year Alarms," Meteorological Applications, Vol. 23, pp. 91-100 (2016).

[31] M. Valipour, M. E. Banihabib, and S. M. R. Behbahani, "Comparison of the ARMA, ARIMA, and the Autoregressive Artificial Neural Network Models in Forecasting the Monthly Inflow of Dez Dam Reservoir," Journal of Hydrology, Vol. 476, pp. 433-441 (2013).

[32] M. Valipour, M. A. G. Sefidkouhi, and S. Eslamian, "Surface Irrigation Simulation Models: A Review," International Journal of Hydrology Science and Technology, Vol. 5, No. 1, pp. 51-70 (2015).

[33] M. Valipour, "Sprinkle and Trickle Irrigation System Design Using Tapered Pipes for Pressure Loss Adjusting," Journal of Agricultural Science, Vol. 4, No. 12, pp. 125-133 (2012)

[34] M. M. Khasraghi, M. A. G. Sefidkouhi, and M. Valipour, "Simulation of Open- and Closed-End Border Irrigation Systems Using SIRMOD," Archives of Agronomy and Soil Science, Vol. 61, No. 7, pp. 929941 (2015).

[35] Q. Yang, D. An, and Y. Cai, "A Novel Evolution Kalman Filter Algorithm for Short-Term Climate Prediction," Asian Journal of Control, Vol. 18, No. 1, pp. 400-405 (2016).

[36] K. Chen, S. Tian, Y. Cheng, and L. Bai, "An Improved MPPT Controller for Photovoltaic System under Partial Shading Condition," IEEE Transactions on Sustainable Energy, Vol. 5, No. 3, pp. 978-985 (2014).

[37] A. Ghaffari, S. Seshagiri, and M. Krstić, "Multivariable Maximum Power Point Tracking for Photovoltaic Micro-Converters Using Extremum Seeking," Control Engineering Practice, Vol. 35, pp. 83-91 (2015).

[38] Y. Yang, H. Li, A. Aichhorn, J. Zheng, and M. Greenleaf, "Sizing Strategy of Distributed Battery Storage System with High Penetration of Photovoltaic for Voltage Regulation and Peak Load Shaving," IEEE Transactions on Smart Grid, Vol. 5, No. 2, pp. 982-991 (2014)

[39] P. Fortenbacher, J. L. Mathieu, and G. Andersson, "Modeling and Optimal Operation of Distributed Battery Storage in Low Voltage Grids," 8 pages (2016), available online at: http://arxiv.org/abs/1603.06468v2

[40] S. Lacey, "How Distributed Battery Storage Will Surpass Grid-Scale Storage in the US by 2020," report in Greentech media, 10 March 2016, available online at: 
www.greentechmedia.com/articles/read/how-distribute d-battery-storage-will-surpass-grid-scale-storage-in-the -us-b

[41] R. O'Regan and A. Mitchell, "Distributed Energy: Small Assets with Big Potential for The UK Electricity Market," report in PWC Energy Spotlight, 13 July 2016, available online at:

http://pwc.blogs.com/energy_spotlight/2016/07/distribu ted-energy-small-assets-with-big-potential-for-uk-elect ricity-market.html

[42] R. O'Regan and A. Mitchell, "Distributed Energy: A Step-Change for Battery Storage in The UK," report in PWC Energy Spotlight, 30 August 2016, available online at:

http://pwc.blogs.com/energy_spotlight/2016/08/distribu ted-energy-a-step-change-for-battery-storage-in-the-uk. html

[43] Project on 2.31-MW PV system with residential area: "Pal Town Josai-no-Mori", in Gunma, Japan (2006), available online at: www.pvdatabase.org/urban_view_details.php?ID $=32$

[44] Project on 2.45-MW PV system with residential area: "Stad van de Zon (City of the Sun)", in Heerhugowaard, Netherlands (2008), available online at: www.pvdatabase.org/urban_view_details.php? $\mathrm{ID}=25$

[45] Project on 2.9-MW PV system with commercial buildings: "PV Canopy in Telefonica - C District", in Madrid, Spain (2006), available online at: pvdatabase.org/projects_view_details.php?ID=298

[46] Top 40 solar beer breweries (2016), available online at: www.solarplaza.com/channels/top-10s/11397/top-40-s olar-beer-breweries/

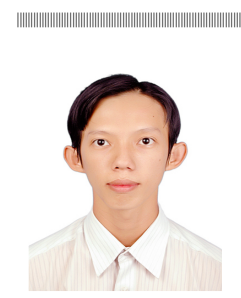

Nguyen Gia Minh Thao obtained B.Eng. (honors) and M.Eng. (research) degrees in Electrical and Electronics Engineering from Ho Chi Minh City University of Technology (HCMUT), Vietnam, in March 2009 and 2011, respectively. He obtained a Dr.Eng. degree in Electrical Engineering from Waseda University, Japan, in 2015. In April 2009, he became a probationary lecturer in the Faculty of Electrical and Electronics Engineering at HCMUT, where he has been a Lecturer since January 2012. He is currently a postdoctoral Research Associate at Waseda University, Japan. His research interests include nonlinear control, intelligent control, renewable energy systems and power network optimization. $\mathrm{He}$ is a member of the Society of Instrument and Control Engineers (SICE) and Asian Control Association (ACA).

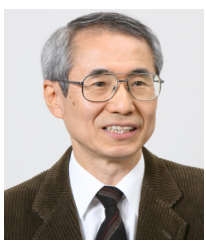

Kenko Uchida received B.S., M.S., and Dr.Eng. degrees in Electrical Engineering from Waseda University, Japan, in 1971, 1973, and 1976, respectively. He is currently a Professor in the Department of Electrical Engineering and Bioscience at Waseda University. His research interests are robust/optimization control and control problems in energy systems and biology. $\mathrm{He}$ is a member of SICE, ACA, the Institute of Electrical Engineers of Japan, and the Institute of Electrical and Electronics Engineers (IEEE). 